OPEN ACCESS

Edited by:

Robert Czajkowski,

University of Gdańsk, Poland

Reviewed by:

Rohit Sharma,

Shoolini University of Biotechnology

and Management Sciences, India

Parisa Gazerani,

Oslo Metropolitan University, Norway

Guilherme Zweig Rocha,

State University of Campinas, Brazil

*Correspondence:

Yanchun Hu

hychun114@163.com

Specialty section:

This article was submitted to

Microbial Symbioses,

a section of the journal

Frontiers in Microbiology

Received: 23 November 2021

Accepted: 04 January 2022

Published: 17 February 2022

Citation:

Okyere SK, Wen J, Cui Y, Xie L, Gao P, Zhang M, Wang J, Wang S, Ran Y, Ren Z and Hu Y (2022) Bacillus toyonensis SAU-19 and SAU-20 Isolated From Ageratina adenophora Alleviates the Intestinal Structure and Integrity Damage Associated With Gut Dysbiosis in Mice Fed High Fat Diet. Front. Microbiol. 13:820236. doi: 10.3389/fmicb.2022.820236

\section{Bacillus toyonensis SAU-19 and SAU-20 Isolated From Ageratina adenophora Alleviates the Intestinal Structure and Integrity Damage Associated With Gut Dysbiosis in Mice Fed High Fat Diet}

Samuel Kumi Okyere', Juan Wen'1, Yujing Cui ${ }^{1}$, Lei Xie', Pei Gao', Ming Zhang', Jianchen Wang ${ }^{1}$, Shu Wang ${ }^{1}$, Yinan Ran ${ }^{1}$, Zhihua Ren ${ }^{1}$ and Yanchun $\mathrm{Hu}^{1,2 *}$

\footnotetext{
1 Key Laboratory of Animal Disease and Human Health of Sichuan Province, College of Veterinary Medicine, Sichuan Agricultural University, Chengdu, China, ${ }^{2}$ New Ruipeng Pet Healthcare Group Co., Ltd., Shenzhen, China
}

This study was performed to identify potential probiotic endophytes from Ageratina adenophora and evaluate their ameliorating effects on gut injury and integrity damage associated with microbiota dysbiosis in mice fed high fat diet. Using morphological and biochemical tests, and 16S rRNA gene sequencing technique, two bacteria endophytes were identified as strains of Bacillus toyonensis and were named Bacillus toyonensis SAU-19 (GenBank No. MW287198) and Bacillus toyonensis SAU-20 (GenBank No. MW287199). Sixty (60) mice were divided into five groups, group 1 was the negative control fed normal diet (NS), group 2 was fed High fat diet (HF), Group 3 was fed High fat diet $+10^{6}$ Lactobacillus rhamnosus (LGG), group 4 was fed High fat $+10^{6}$ Bacillus toyonensis SAU-19 and group 5 fed High fat diet $+10^{6}$ Bacillus toyonensis SAU-20. After 35 days, histological and immunohistochemistry examination were performed in the ileum tissues. Furthermore, DAO and antioxidants activities were measured in serum, mRNA expressions of tight junction proteins (occludin and ZO-1) and inflammation related cytokines (IL-1 $\beta, T F N-\alpha, I L-2, I L-4$, and IL-10) in the ileum tissues as well as slgA levels and total bacteria (Escherichia coli, Salmonella, Staphylococcus, and Lactobacillus) in the small intestine and cecum content. The results showed an increase in the DAO activity, oxidative stress parameter (MDA), pro-inflammation cytokines (IL$1 \beta, T F N-\alpha, I L-2)$, reduce immunity (slgA), and destroyed intestinal structure and integrity (reduce tight junction proteins) in the high fat diet group and this was associated with destruction of the gut microbiota composition (increasing pathogenic bacteria; E. coli, Salmonella, Staphylococcus and reducing beneficial bacteria, Lactobacillus spp.) in mice $(P<0.05)$. However, the administration of Bacillus toyonensis SAU-19 and SAU20 reverted these effects. Our findings indicated that, Bacillus toyonensis SAU-19 and 
SAU-20 isolated from $A$. adenophora could prevent the excess weight gain from high fat diet feeding, improved antioxidant status and alleviated the intestine integrity damage as well as reduce the population of enteric bacteria such as E. coli, Salmonella, and $S$. aureus and increasing the population of beneficial bacteria such as Lactobacillus in the gut of mice fed high fat diet, therefore, can serve as a potential probiotics in humans and animals.

Keywords: Ageratina adenophora, endophytes, Bacillus toyonensis SAU-19, Bacillus toyonensis SAU-20, intestinal integrity, tight junction proteins, inflammation cytokines

\section{INTRODUCTION}

Probiotics are live microorganisms that, when administered in adequate amounts, confer health benefits on the host (FAO/WHO, 2002). Probiotics competitively occupy the receptors on mucosal epithelial cells and inhibit the adhesion of pathogens to epithelial walls (Miljkovic et al., 2015). Probiotics have been reported to improve intestinal structure, integrity and microbiota (Wang et al., 2020; Yu et al., 2020; Sudan et al., 2021). Over the past years, probiotics were mainly isolated from milk sources, however with the high demand for non-animal probiotic products by vegetarians, isolation and development of probiotics from plant sources is on the rise (Nematollahi et al., 2016).

The internal tissues of plants harbor a class of beneficial endosymbiotic microorgan-isms (predominantly bacteria and fungi) called endophytes (Kumara et al., 2014). In this plantendophyte relationship, plants offer nourishment and protection to the endophytes, whereas, endophytes enhance defense, health, and stress tolerance of plants (Kandel et al., 2017). Numerous studies have isolated several endophytes with probiotics potential from fruits, leaves and other plant parts (Touret et al., 2018; Pabari et al., 2020; Ragul et al., 2020; Unban et al., 2020).

The Asteraceae plant, Ageratina adenophora (Spreng) R. M. King et $\mathrm{H}$. Rob. is the most destructive invasive plant that threatens the native biodiversity in the southwestern regions of China (Sun et al., 2018). In addition, studies have indicated that, this plant induces toxicity in both humans and animals (Ouyang et al., 2014; He et al., 2016; Mo et al., 2017; Okyere et al., 2020; Ren et al., 2021a,b). Currently, research on A. adenophora has gradually moved from toxicity prevention to biological utilization of the plant in vivo (Xu et al., 2018; Chen L. et al., 2020). Due to the invasive nature and growth performance of this plant, some scientists are focusing on harnessing the chemical substances and other products in the plant's resources and developing them into useful products (Fu et al., 2019; Okyere et al., 2021). Therefore, we hypothesized that, $A$. adenophora may contain endophytes that have potential probiotic activity.

High fat diets cause a shift in gut bacterial community and a modest increase in circulating endotoxin concentrations (Cani et al., 2007). The dysbiosis of the gut microbiota may results in disturbances in the normal physiological processes of the body, hence, may affect the overall mice growth performance and gut integrity via increasing the population of harmful bacteria and viruses which invade tissues and produce lethal toxins and metabolites to damage them (Abaidullah et al., 2019).
Thus, this study was performed for the first time to isolate, characterize and identify potential probiotic endophytes from A. adenophora plant and evaluate its ameliorative effects (in vivo) on intestinal structure and integrity damage, and gut microbiota dysbiosis induce by high fat diet in mice. This study will give the basis for further investigation and future development of probiotics and identification of other beneficial endophytes from $A$. adenophora, thereby utilizing the plant resource for human and animal benefit.

\section{MATERIALS AND METHODS}

\section{Sample Collection}

Leaves and stems of $A$. adenophora were collected from Wangsuo Village, Cangzhou Street, Dechang County, Liangshan Yi Autonomous Prefecture, Sichuan Province $\left(102^{\circ} 15^{\prime} 20^{\prime \prime} \mathrm{E}\right.$ and $27^{\circ} 20^{\prime} 11^{\prime \prime} \mathrm{N}$; elevation $\left.=2152 \mathrm{~m}\right)$. The samples were confirmed as A. adenophora by Prof. Chao Hu, Department of Botany, Sichuan Agricultural University. Culture media were purchased from Qingdao Hope Bio-Technology Co., Ltd., Qingdao, China. Mice, basal and high fat diet (Table 1) were purchased from Chengdu Dashuo Experiment Animal Co. Ltd., Chengdu, China. E. coli CMCC44103, Salmonella typhimurium ST19, and Lactobacillus rhamnosus GG ATCC 53103 was obtained from the College of Veterinary Medicine (Professor Xueqin Ni's lab), Sichuan Agricultural University, China.

\section{Plant Samples Sterilization}

Leaf and stem were separated from the plant and washed thoroughly under running tap water to remove adhering soil

TABLE 1 | Composition of the basal diet.

\begin{tabular}{|c|c|c|c|}
\hline \multirow{2}{*}{$\frac{\text { Normal diet }}{\text { Ingredients }}$} & \multirow[b]{2}{*}{ Content g/kg } & \multirow{2}{*}{$\frac{\text { High fat diet }}{\text { Ingredients }}$} & \multirow[b]{2}{*}{ Content $\mathrm{g} / \mathrm{kg}$} \\
\hline & & & \\
\hline Water & 94 & Water & 93 \\
\hline Protein & 190 & Protein & 134 \\
\hline Fat & 51 & Fat & 143 \\
\hline Fiber & 36 & Fiber & 27 \\
\hline Ash & 62 & Ash & 44 \\
\hline Calcium & 11.3 & Calcium & 8.3 \\
\hline Phosphorus & 8.6 & Phosphorus & 7.1 \\
\hline
\end{tabular}


particles and microbes. The surface decontamination method described in a previous study was used with some minor modification (Fisher et al., 1992). Leaf and stem samples were further cleaned with detergent (5\% Teepol), under running tap water and then distilled water. The cleaned samples were then placed in a laminar hood and immersed in 70\% ethanol and sodium hypochlorite (5\%) solution for $30 \mathrm{~s}$ and 3 min respectively. The samples were further exposed to absolute alcohol for $10 \mathrm{~s}$, thereafter; they were washed with autoclaved distilled water three times. Surface decontamination was performed on the samples after cutting them into pieces $\left(1 \mathrm{~cm}^{2}\right)$ with sterile surgical blade.

\section{Isolation and Purification of Potential Probiotic Endophyte}

The isolation of potential probiotic endophytes was conducted following the procedures described in a previous study (Bhore et al., 2010). Well grown colonies of cultivable bacterial putative-endophytes that were visible on the LB Agar were randomly selected for further experiments. Pure cultures of each putative endophyte were cultivated separately in universal bottles containing $10 \mathrm{~mL} \mathrm{LB}$ broth. Culture-cultivation was carried out at $37^{\circ} \mathrm{C}$ for $20 \mathrm{~h}$. Glycerol stocks (30\%) were prepared and kept at $-80^{\circ} \mathrm{C}$ to preserve the putative probiotic endophytes for subsequent research.

\section{Preliminary Identification of Potential Probiotic Endophyte}

Distinctive colonies on LB agar plates were subjected to preliminary identification using colony characteristics, Gram staining and catalases activity.

Colony characteristics such as color, elevation, margins and surfaces of each putative probiotic endophyte were studied using magnifying lens.

\section{Gram Staining}

Gram staining of the isolates was performed using commercial kits (Nanjing Duly Biotech Co., Ltd., Jiangsu, China) following the manufacturer's instructions.

\section{Catalase Test}

Pure colonies of isolates were placed on a slide, and then a drop of hydrogen peroxide (3\%) was added to the isolates on the slides. The formation of bubbles showed a catalase positive result, whereas absence of bubbles showed a catalase negative reaction.

Only colonies showing phenotypic features similar to lactic acid bacteria such as gram-positive, catalase-negative reaction, and white or creamy colony color were selected for further studies. We further performed a justification experiment to substantiate the probiotic potential of endophytes through other morphological, biochemical and probiotic tests. These tests included bacteria size, shape and arrangement determination, $\mathrm{NaCl}$, acid and bile salt tolerance, glucose fermentation, antimicrobial activity and antibiotic sensitivity. All tests were performed in triplicate.

\section{Morphological and Biochemical Characterization of Potential Probiotic Endophyte}

The size, shape and arrangement of each putative probiotic endophyte were observed using an electronic microscope (INB129 USB Digital Electronic Operation Microscope Driver, Guangzhou, China) (Ahmad and Zargar, 2017).

The isolates were biochemically characterized using glucose, sucrose and D-sorbitol fermenting test, and sodium chloride $(\mathrm{NaCl})$ inhibition test (Zaved et al., 2008).

\section{Carbohydrate Fermentation Test}

Glucose, sucrose and D-sorbitol fermentation were conducted in LB fermentation broth with $0.004 \%$ phenol red containing $2 \%$ of glucose, sucrose, and D-sorbitol. All the tubes were sterilized for $15 \mathrm{~min}$ at $121^{\circ} \mathrm{C}$. The tubes were inoculated with a single colony of bacteria. The positive reaction of the bacteria was indicated by the changes in the color of the medium (from red to yellow).

\section{Tolerance to Inhibitory Substances Test}

To observe the $\mathrm{NaCl}$ tolerance of the isolates, $1 \%$ fresh overnight culture of the isolates was incubated in LB broth with 0, 2, 4, 6.5\% $\mathrm{NaCl}$ concentration for $24 \mathrm{~h}$. The turbidity in broth was observed as $\mathrm{NaCl}$ tolerance (Chakraborty and Bhowal, 2015). Broth with no bile salt was used as a positive control whereas broth with no bile salt and isolate was used as the negative control.

\section{Probiotic Characterization of Potential Probiotic Endophyte}

Optimization of Growth Parameters (pH)

LB broths with different pH's were prepared with 1M HCL and $1 \mathrm{M} \mathrm{NaOH}$. The isolated endophyte cultures were inoculated into sterile LB broth tubes of varying $\mathrm{pH}$ (i.e., $\mathrm{pH} 2.5$, 4.5, and 6.5) and incubated at $37^{\circ} \mathrm{C}$ for $24 \mathrm{~h}$. The turbidity of the medium was observed to be $\mathrm{pH}$ tolerant (Tambekar and Bhutada, 2010).

\section{Bile Salt Tolerance}

For the bile salt tolerance test, LB medium with varying concentrations $(0,0.2$, and $0.4 \%)$ of bile salt [sodium deoxycholate (DCA)] were inoculated with selected endophyte cultures and incubated at $37^{\circ} \mathrm{C}$ for $24 \mathrm{~h}$. The turbidity of the medium was observed to be bile salt tolerance (Tambekar and Bhutada, 2010). Broth with no bile salt was used as positive control whereas broth with no bile salt and isolate was designated as the negative control.

\section{Antibiotic Sensitivity Test}

Antibiotic sensitivity patterns were evaluated using the commercial kit method (Oxoid Thermo Fisher Biochemicals Ltd., Beijing, China). Different types of common antibiotic disks were loaded onto the surface of the medium and kept at room temperature for $10 \mathrm{~min}$. After that, the plates were incubated at $37^{\circ} \mathrm{C}$ for $24 \mathrm{~h}$. The diameters of the zones of inhibition were measured in millimeters using a Vernier caliper. The antibiotics tested were tetracycline $(30 \mu \mathrm{g})$, erythromycin $(15 \mu \mathrm{g})$, sultamicillin $(10 \mu \mathrm{g})$, ampicillin $(20 \mu \mathrm{g})$, and streptomycin 
(10 $\mu \mathrm{g})$. Disks immersed in sterile water were used as the negative controls.

Inhibition zones were characterized based on inhibition diameter as resistant ( $\mathrm{R}$ ) (zone $<10 \mathrm{~mm}$ ), moderately susceptible (MS) (zone 10-20 mm), susceptible (S) (zone $>20 \mathrm{~mm}$ ), and very susceptible (VS) (zone $>31 \mathrm{~mm}$ ) (Talib et al., 2019).

\section{Antimicrobial Activity}

The tested organisms E. coli CMCC44103 and Salmonella typhimurium ST19 were maintained in their respective broths in screw-capped tubes and kept at $4^{\circ} \mathrm{C}$. The tested microorganisms were standardized using $0.5 \mathrm{McF}$ arland standards (cell density of $1.5 \times 10^{8} \mathrm{CFU} / \mathrm{mL}$, with absorbance of 0.132 at a wavelength of $600 \mathrm{~nm}$ ) (Talib et al., 2019).

For screening of endophyte cultures, these cultures were inoculated in $\mathrm{LB}$ broth and incubated at $37^{\circ} \mathrm{C}$ for $24 \mathrm{~h}$ on a shaker to carry out the fermentation process. After incubation, $1 \mathrm{~mL}$ of each fermented culture broth was used to test the antimicrobial activity against the two pathogenic bacteria by agar well diffusion method. The endophyte culture was screened against E. coli CMCC44103 and Salmonella typhimurium ST19. Overnight cultures of pathogens grown in their respective medium at $37^{\circ} \mathrm{C}$ were diluted to a turbidity equivalent to that of a $0.5 \mathrm{McFarland}$ standard (Khunajakr et al., 2008). The inoculum was spread over the surface of petri dishes containing LB agar (Difco); and then wells were perforated in the agar in which culture supernatants of the selected endophyte was added. Sterile water was used as the control. The plates were then incubated for $24 \mathrm{~h}$ at $37^{\circ} \mathrm{C}$ in a $5 \% \mathrm{CO}_{2}$ atmosphere. After incubation, the presence of inhibition halos with diameters $>1 \mathrm{~mm}$ were considered as positive results (Pessoa et al., 2017).

\section{S rRNA Gene Sequencing of Selected Potential Probiotic Endophyte}

The DNA's of the selected endophytes were extracted using the commercial kit method (QlAamp DNA mini kit-Qiagen). The 16S rRNA was amplified by PCR (Applied Biosystems9700) using universal primers (27F AGA GTT TGA TCM TGG CTC AG and 1492R GTATTA CCGCGGCTG CTG G) and sequenced using a Genetic Analyzer 3130 (Applied Biosystems, Wakefield, United States) in Solgent Co. Ltd. (16S rRNA, Seoul, South Korea). The aligned 16S rRNA of the isolates were subjected to BLAST using the non-redundant database of NCBI Genbank. Based on the maximum identity score $(99 \%$ and above) they were selected and aligned using Clustal W multiple alignment software. Considering the results of sequence analyses, a phylogenetic tree was formed via the neighbor-joining method using the software package MEGA X (Jinglong et al., 2019).

\section{Animal (in vivo) Experiment Experimental Animals}

A total number of 72 (5 weeks old; BW 25-30 g) live and apparently healthy male Specific-pathogen free) Kun ming mice (SPFKM) mice were used in this study. Twelve (12) mice were used for the pilot experiment, and 60 mice were used in the feeding experiment. For the pilot experiment, mice were allocated equally into three groups; the control group was fed normal diet and $1 \mathrm{ml}$ of $0.9 \%$ normal saline in drinking water while the other groups were fed normal diet and $1 \mathrm{ml}$ of $1 \times 10^{6}$ (minimum probiotic consumption range) (Kechagia et al., 2013) of selected endophyte suspension in drinking water for 20 days at a controlled temperature (range $22-24^{\circ} \mathrm{C}$ ) and humidity (range 40-60\%) room. Mortality, illness (such as diarrhea) and feed intake were recorded each day until the day 20. The results indicated that daily administration of both endophytes did not reduce feed intake or cause any illness and mortality, hence it was safe for use in the experiment. This study was approved by the Institutional Animal Care and Use Committee of Sichuan Agricultural University, Sichuan, China, under the permit number DKY-B2019603005. The study was also performed in accordance with the ARRIVE guidelines ${ }^{1}$.

\section{Commercial Probiotic}

Lactobacillus rhamnosus GG ATCC 53103 is a commercial product being used all over the world. It was originally isolated from feces of a healthy human adult by Professor Xueqin Ni's lab in College of Veterinary Medicine, Sichuan Agricultural University, Chengdu, China. This bacterial strain improved the growth and health of poultry and large animals at a safe dose range of $1 \times 10^{6}-1 \times 10^{11} \mathrm{CFU} / \mathrm{ml}$ or $\mathrm{CFU} / \mathrm{g}$. In addition, the beneficial effects of this strain have been widely studied in both clinical and human intervention studies (Segers and Lebeer, 2014), hence we selected and used it as a bench mark for comparing our isolates effectiveness and safety in this study. We obtained pure samples and made fresh samples for administration each week and stored them in a refrigerator $\left(4^{\circ} \mathrm{C}\right)$ until the 35 days of the experiment were finished.

\section{Experimental Design}

Sixty (60) male mice (5 weeks old; BW 25-30 g) were randomly allocated into five groups; 12 mice per group, and 4 mice per replicate in each group. Group A (negative control) was fed a normal diet $(20 \mathrm{~g}$ normal diet $)+1 \mathrm{~mL}$ daily amounts of $0.9 \%$ normal saline (NC), Group B (positive control) was fed a High fat diet $(20 \mathrm{~g})+1 \mathrm{~mL}$ daily amounts of $0.9 \%$ normal saline (HF), Group C was fed High fat diet $(20 \mathrm{~g})+1 \mathrm{~mL}$

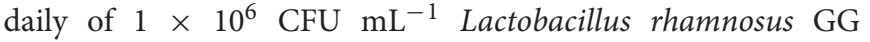
suspension (HF + LGG), Group C was fed a High fat diet $(20 \mathrm{~g})+1 \mathrm{~mL}$ of $1 \times 10^{6} \mathrm{CFU} \mathrm{mL}^{-1}$ Bacillus toyonensis SAU19 (HF + B. toyo. SAU-19) and Group D fed with High fat diet $(20 \mathrm{~g})+1 \mathrm{~mL}$ of $1 \times 10^{6} \mathrm{CFU} \mathrm{mL}^{-1}$ Bacillus toyonensis SAU-20 $(\mathrm{HF}+$ B. toyo. $S A U-20)$ suspension in drinking water for 35 days. Experimental duration was selected according to our previous work (unpublished) which showed that 35 days of feeding of high fat diet could cause gut dysbiosis and the work by Delgado et al. (2009) and Xin et al. (2020). The administration of both the high fat diet and putative probiotics were initiated the same time in this study. The mice were housed in cages at the departmental experimental house and were exposed to a $12 \mathrm{~h}$ cycle of light and darkness. The experiment was carried out in a temperature (range $22-24^{\circ} \mathrm{C}$ ) and humidity (40-60\%) controlled environment. The animals were monitored daily for any abnormality, illness (such as diarrhea) and death. Throughout the experiment, at exactly

${ }^{1}$ http://www.nc3rs.org.uk/arrive-guidelines 
8:00am, normal (NS), high fat diet, high fat diet + commercial probiotic (NP) and high fat diet + selected potential probiotic endophytes were supplemented to their respective groups. Feed intake was monitored and recorded daily throughout the experimental period. Clean water was provided ad libitum in clean water bottles. Water bottles were washed every week and fresh drinking water was placed in it for the next week's administration. The bedding material (wood shavings) was also changed weekly. To administer the potential probiotic endophyte and LGG, new stocks were generated each week in LB and MRS broths and their viability was monitored by serial dilution and viable cell count using LB and MRS agar respectively.

After the 35-day experimental period, the general health, weight gain, and feed conversion ratio were measured. Subsets of the mice were euthanized by $\mathrm{CO}_{2}$ asphyxiation on $(n=8$ mice/group); all mice were fasted for $12 \mathrm{~h}$ prior to being euthanized. At necropsy, blood from the abdominal aorta was collected into anticoagulant-coated tubes; and the small intestine was then aseptically removed and processed as indicated in the assays outlined below.

\section{Preparation of Bacterial Suspension}

Potential probiotic endophytes were transferred twice into LB broths and incubated anaerobically at $37^{\circ} \mathrm{C}$ for $72 \mathrm{~h}$. The bacterial cells were collected by centrifugation (3,500 g, $5 \mathrm{~min}$ ), and washed twice in $0.85 \% \mathrm{NaCl}$ (Sigma), and then resuspended in $0.85 \%$ $\mathrm{NaCl}$ to a final concentration of $1 \times 10^{6} \mathrm{CFU} / \mathrm{mL}$ and stored at $4^{\circ} \mathrm{C}$.

\section{Histopathological Analysis}

After weighing the isolated intestine, the ileum section was cut aseptically and samples (1/2 of each intestine collected at necropsy) were fixed in $10 \%$ buffered neutral formalin solution, and then dehydrated in graded (70-100\%) alcohol. After being cleared in xylene, each sample was embedded in paraffin and $5 \mu \mathrm{m}$-thick sections were prepared. For analyses, samples were stained with hematoxylin and eosin (H\&E) dye and then evaluated using a light microscope. A total of eight mice per group were evaluated; for each mouse, four stained slices were examined. Two independent investigators who were blinded to the treatment evaluated the slides. A 0-3 point scale was used to describe the severity of inflammation $(0=$ none, $1=$ mild, $2=$ moderate, and $3=$ severe). Each parameter was calculated and summed to obtain the overall score (Jang et al., 2019; Fan et al., 2021). Slides were also blinded, and Image Pro Plus 6.0 (Media Cybernetics, Bethesda, MD, United States) software was used to measure villus height and crypt depth. A villus was considered suitable to measure if it was completely intact and had two intact crypts on both sides. Suitable crypts were those that were intact and flanked by two intact villi.

\section{Determination of Diamine Oxidase Levels in Serum of Mice}

The concentration of diamine oxidase (DAO) was determined in the blood serum using commercial ELISA kits (Jingmei Biological Technology, Jiangsu, China) to assess the degree of gut mucosal barrier damage, according to the manufacturer's instructions. The level of sensitivity of each kit was $0.1 \mathrm{pg} / \mathrm{ml}$.

\section{Determination of Antioxidant Capacity in Serum of Mice}

Antioxidant indexes in serum at 35 days were measured using commercial kits (Nanjing Jiancheng Bioengineering Institute, Nanjing, Jiangsu, China) following the manufacturer's instructions. The antioxidant parameters checked were catalase (CAT), superoxide dismutase (SOD), glutathione (GSH), and malondialdehyde (MDA).

\section{Determination of Secretory Immunoglobulin A Levels in Small Intestine Contents of Mice}

Fresh small intestine contents of two mice from each replicate were sampled in 5-ml microcentrifuge tubes at day 35 and stored at $-80^{\circ} \mathrm{C}$. ELISA kits (Sagon Biotech, Shanghai, China) were used to enumerate Secretory Immunoglobulin A ( $\operatorname{IgA}$ ) levels in the small intestine contents following the manufacturer's instructions. The level of sensitivity of the kit was $0.1 \mu \mathrm{g} / \mathrm{ml}$.

\section{Immunohistochemistry Assay}

The small intestine was carefully dissected from the mice, washed with cold PBS ( $\mathrm{pH} \mathrm{7.2-7.4)} \mathrm{and} \mathrm{then} \mathrm{fixed} \mathrm{overnight}$ in $4 \%$ paraformaldehyde, and embedded in paraffin wax after dehydration. The paraffin-embedded intestinal tissues were sliced into $4 \mu \mathrm{m}$ sections and then dewaxed in xylene, followed by rehydrating through a graded series of ethanol solutions. Endogenous peroxidase was blocked by incubating with $3 \%$ $\mathrm{H}_{2} \mathrm{O}_{2}$ in methanol for $10 \mathrm{~min}$. After, heat-induced antigen retrieval was performed using citrate buffer $(10 \mathrm{mM}, \mathrm{pH}=6.0)$. The sections were blocked with goat serum (Sigma, St. Louis, MO, United States) for $15 \mathrm{mins}$, and incubated overnight at $4^{\circ} \mathrm{C}$ with a rabbit monoclonal anti-mouse occludin antibody (ab216327, Abcom, dilution 1:200). Each section was rewarmed at $37^{\circ} \mathrm{C}$ for $1 \mathrm{~h}$ and washed in PBS for $10 \mathrm{~min}$, followed by incubation with biotin-tagged anti-rabbit second antibody (SP9001, ZSGB-BIO, Beijing, China) at $25^{\circ} \mathrm{C}$ for $15 \mathrm{mins}$. Furthermore, horseradish peroxidase-labeled streptavidin working solution (S-A/HRP) was added and after $15 \mathrm{~min}$, the mixture was slowly flushed with PBS. Finally, freshly prepared DAB chromogenic solution was added, then after $6 \mathrm{~min}$, hematoxylin was also added for $20 \mathrm{~s}$. The slides were examined for positive staining and were subjected to optical density analysis with a Leica DM1000 microscope (Leica Microsystems Imaging Solutions Ltd., Cambridge, United Kingdom) using Image Pro Plus 6.0 (Media Cybernetics, Bethesda, MD, United States), three visual fields were randomly selected in each sections.

\section{Extraction of Total RNA and Quantitative Real Time Polymerase Chain Reaction}

Samples of small intestine tissues (30 mg/mouse) were snapfrozen with liquid $\mathrm{N}_{2}$ and then immediately ground into powder using a ceramic mortar. Total RNA from each sample was extracted using an Animal Total RNA Isolation Kit 
(Sagon Biotech, Shanghai, China) according to manufacturer's instructions. After confirming the isolated RNA concentration and purity using a NanoDrop One system (Thermo Fisher Scientific, Waltham, MA, United States; OD260/280 $\approx 1.9-$ 2.0 ), triplicate aliquots (each $1 \mu \mathrm{g}$ ) were removed, loaded into wells, and cDNA was prepared using a PrimeScrip RT reagent kit (Takara, Tokyo, Japan). Thereafter, quantitative real time polymerase chain reaction (qRT-PCR) was performed using a SYBR Premix ExTaq (Takara, Tokyo, Japan) and a CFX96 thermal cycler (Bio-Rad, Hercules, CA, United States). The PCR conditions were shown as follows: $95^{\circ} \mathrm{C}$ for $5 \mathrm{~min}$, followed by 40 cycles of $95^{\circ} \mathrm{C}, 15 \mathrm{~s}$ for denaturation, $60^{\circ} \mathrm{C}, 60 \mathrm{~s}$ for annealing at and $70^{\circ} \mathrm{C}, 25 \mathrm{~s}$ for extension. Each $\mathrm{qRT}-\mathrm{PCR}$ reaction was performed with volumes of $10 \mu \mathrm{L}$ containing $5 \mu \mathrm{L}$ TB Green TM Premix (Takara), $1 \mu \mathrm{L}$ forward and reverse primers, $1 \mu \mathrm{L}$ cDNA, and $2 \mu \mathrm{L}$ DNase/RNase-Free Deionized Water (Tiangen, Beijing, China). The primers used to analyze the genes of interest were designed from NCBI genBank and are shown in Table 2. Relative gene expression in each sample was normalized to an internal control ( $\beta$-actin); data analysis was performed using the $2^{-\Delta \Delta}$ $\mathrm{Ct}^{\mathrm{C}}$ method. All samples were evaluated in triplicate.

\section{Quantification of Small Intestine and Cecum Microbial Content in Mice}

At the end of the experimental period, eight mice from each group were sacrificed humanly and then, small intestine and cecum contents were aseptically collected into sterile tubes and stored at $-80^{\circ} \mathrm{C}$ for further analysis. An aliquot of fresh small intestine and cecum contents $(1 \mathrm{~g})$ were diluted in sterile saline solution at a ratio of 1:10. Diluted contents were vortexed for $2 \mathrm{~min}$ to obtain a homogeneous suspension, thereafter, $0.1 \mathrm{~mL}$ was spread on different culture media for total bacterial count. CHROMagar agar (Qingdao Hope Bio-Technology Co., Ltd., Qingdao, China) was used to enumerate E. coli, bismuth sulfite agar (Qingdao Hope Bio-Technology Co., Ltd., Qingdao, China), for Salmonella enumeration, mannitol salt agar (Qingdao Hope
Bio-Technology Co., Ltd., Qingdao, China) for Staphylococcus aureus whereas De Man Rogosa Sharpe agar (Guangzhou Ikeme Technology Co., Ltd., Guangzhou, China) was used for the growth and enumeration of Lactobacillus species. The plates were incubated under anaerobic conditions at $37^{\circ} \mathrm{C}$ for $24 \mathrm{~h}$. Furthermore, colonies obtained on the plates were counted and microbial populations were expressed as $\log _{10} \mathrm{CFU} / \mathrm{g}$ of small intestine and cecum contents.

\section{Statistical Analysis}

Statistical analysis of the data collected (from various independent experiments) was performed using GraphPad Prism 5.04 software (GraphPad Software, Inc., La Jolla, CA, United States) and SPSS 20 Statistical Analysis Software (SPSS Inc., Chicago, IL, United States). The Shapiro-Wilk Test was used to test the normality of the data. The experimental data were first tested by normal distribution, and on this basis, we carried out one-way analysis of variance (ANOVA), in which the statistics included the homogeneity test of variance. All experimental results are presented as mean $\pm \mathrm{SD}$, and statistical significance were determined by one-way analysis of variance (ANOVA) followed by the Tukey's test. The values were significantly different at $P<0.05$.

\section{RESULTS}

From the 13 distinctive bacteria colonies on the LB agar of both cultured leaves and stems of A. adenophora, two colonies were selected based on the results of the preliminary (colony color, gram staining, and catalase test), morphology (bacteria size, shape, and arrangements) and biochemical ( $\mathrm{NaCl}$ tolerance) tests. The two endophytes (one from leaf and another from the stem labeled as LB60 and LB68 respectively) were gram positive, creamy white, $\mathrm{NaCl}$ tolerant and catalase negative which were basic characteristics for bacteria

TABLE 2 | Primers used for the real-time PCR analysis.

\begin{tabular}{|c|c|c|c|c|c|}
\hline Gene name & Primer & Sequence $\left(5^{\prime}\right.$ and $\left.3^{\prime}\right)$ & Product length (bp) & Annealing Temperature $\left({ }^{\circ} \mathrm{C}\right)$ & Sequence number \\
\hline $\mid \mathrm{L}-1 \beta$ & Reverse & ATGTGCTGCTGCGAGATTTG & & & \\
\hline IL-2 & Reverse & AAAGTCCACCACAGTTGCTG & & & \\
\hline \multirow[t]{2}{*}{ IL-4 } & Forward & GTACCAGGAGCCATATCCACG & 130 & 60.18 & NM_021283.2 \\
\hline & Reverse & TTCGTTGCTGTGAGGACGTT & & & \\
\hline IL-10 & Reverse & GCAGAGGAGGTCACACCATTT & & & \\
\hline \multirow[t]{2}{*}{ TNF- $\alpha$} & Forward & СССТСАСАСТСАСАAАССАС & 211 & 59.82 & NM_001278601.1 \\
\hline & Reverse & ATAGCAAATCGGCTGACGGT & & & \\
\hline \multirow[t]{2}{*}{ Ocln } & Forward & ССТССАСССССАТСТGАСТА & 79 & 60.03 & NM_001360536.1 \\
\hline & Reverse & GCTTGCCATTCACTTGCCA & & & \\
\hline ZO-1 & Forward & CCTGACGGTTGGTCTITGC & 114 & 59.69 & NM_001163574.1 \\
\hline
\end{tabular}


to be classified as a probiotic (Alfonzo et al., 2016), hence were selected for further probiotic test $(\mathrm{pH}$, and bile salt tolerance, antibiotic sensitivity, antimicrobial activity, and sugar fermentation), identified (using $16 \mathrm{~S}$ rRNA) and used for the animal experiment.

\section{Morphological and Biochemical Characterization}

The morphological characteristics of the selected endophytes (LB60 and LB68) are shown in Table 3.

The results of the catalase test are listed in Table 3. Both LB60 and LB68 endophytes produced no bubbles with $3 \% \mathrm{H}_{2} \mathrm{O}_{2}$ (catalase negative).

In this study, a change in the culture medium from red to yellow gave an indication of acid production during carbohydrate fermentation test. LB68 could change the color

TABLE 3 | Preliminary, morphological, and biochemical characteristics of LB60 and LB68 bacteria isolated from Ageratina adenophora.

\begin{tabular}{|c|c|c|c|}
\hline & Parameters & LB68 & LB60 \\
\hline & $\begin{array}{l}\text { Part of plant } \\
\text { isolated }\end{array}$ & Stem & Leaf \\
\hline & Size & $1 \mathrm{~mm}$ & $2 \mathrm{~mm}$ \\
\hline \multirow[t]{5}{*}{ Characteristics } & $\begin{array}{l}\text { Culture media } \\
\text { characteristics }\end{array}$ & $\begin{array}{l}\text { Colonies round, } \\
\text { creamy, smooth, } \\
\text { convex, moist, raised } \\
\text { and entire }\end{array}$ & $\begin{array}{c}\text { Colonies irregular, } \\
\text { creamy, mucoid, } \\
\text { opaque, raised and } \\
\text { wavy }\end{array}$ \\
\hline & $\begin{array}{l}\text { Isolates } \\
\text { morphology }\end{array}$ & Singles rods & $\begin{array}{c}\text { Rods in chains ( } 2-3 \\
\text { pairs) }\end{array}$ \\
\hline & Gram staining & Positive (+) & Positive (+) \\
\hline & Catalase & Negative (-) & Negative $(-)$ \\
\hline & Glucose & Positive (+) & Positive (+) \\
\hline \multirow[t]{2}{*}{$\begin{array}{l}\text { Sugar } \\
\text { fermentation }\end{array}$} & $\begin{array}{l}\text { Sucrose } \\
\text { D-sorbitol }\end{array}$ & $\begin{array}{l}\text { Positive (+) } \\
\text { Negative (-) }\end{array}$ & $\begin{array}{l}\text { Positive (+) } \\
\text { Positive (+) }\end{array}$ \\
\hline & 2.5 & ++ & ++ \\
\hline \multirow[t]{3}{*}{ Varying $\mathrm{pH}$} & 4.5 & ++ & ++ \\
\hline & 6.5 & ++ & ++ \\
\hline & 0 & ++ & ++ \\
\hline \multirow{3}{*}{$\begin{array}{l}\text { Bile salt } \\
\text { concentration } \\
(\%)\end{array}$} & 0.2 & ++ & ++ \\
\hline & 0.4 & ++ & ++ \\
\hline & 0 & ++ & ++ \\
\hline \multirow{5}{*}{$\begin{array}{l}\mathrm{NaCl} \\
\text { concentration } \\
(\%)\end{array}$} & 2 & ++ & ++ \\
\hline & 4 & ++ & ++ \\
\hline & 6.5 & ++ & ++ \\
\hline & $\begin{array}{l}\text { Streptomycin } \\
(10 \mu \mathrm{g})\end{array}$ & $12^{\mathrm{MS}}$ & $22^{S}$ \\
\hline & Tetracycline $(30 \mu \mathrm{g})$ & $21^{\mathrm{S}}$ & $28^{S}$ \\
\hline \multirow[t]{4}{*}{ Antibiotics } & $\begin{array}{l}\text { Erythromycin } \\
\qquad(15 \mu \mathrm{g})\end{array}$ & $-^{R}$ & $8^{\mathrm{R}}$ \\
\hline & Ampicillin $(20 \mu \mathrm{g})$ & $-^{R}$ & $-{ }^{R}$ \\
\hline & Sultamicillin $(10 \mu \mathrm{g})$ & $30^{\mathrm{S}}$ & $25^{\mathrm{S}}$ \\
\hline & $\begin{array}{c}\text { E. coli } \\
\text { CMCC44103 }\end{array}$ & - & - \\
\hline Antibacterial & $\begin{array}{c}\text { Salmonella } \\
\text { typhimurium S19 }\end{array}$ & - & - \\
\hline
\end{tabular}

++ , Turbidity of the growth medium. Resistant $(R)$ (zone $<10 \mathrm{~mm}$ ), moderately susceptible (MS) (zone 10-20 mm) and susceptible (S) (zone $>20 \mathrm{~mm}$ ) and very susceptible (VS) (zone > $31 \mathrm{~mm}$ ). "-" No zone of inhibition on growth medium. of the medium for both glucose and sucrose but not Dsorbitol; however, LB60 changed the medium for all tested sugars (Table 3).

Furthermore, the $\mathrm{NaCl}$ tolerance test after $24 \mathrm{~h}$ showed that, both endophytes (LB60 and LB68) showed growth in broths of all tested $\mathrm{NaCl}$ concentrations (Table 3 ).

\section{Probiotic Characteristics Test}

From the results, both endophytes (LB60 and LB68) showed growth in all the broths at different $\mathrm{pH}$ and bile salt concentrations (Table 3). Moreover, the antibiotic resistance test revealed that both $\mathrm{LB} 60$ and LB68 were susceptible to streptomycin, tetracycline and sultamicillin but resistant to erythromycin and ampicillin (Table 3). In addition, results from the antimicrobial test showed no zone of inhibition on agar of the tested pathogenic bacteria for either endophyte (Table 3).

\section{Identification of LB60 and LB68}

Two strains, LB60 and LB68, were screened from 13 colonies for further evaluation based on their performance on LB agar plates. In the assay, LB60 and LB68 showed the largest clearing zones; isolation was carried out based on the appearance of LAB and the clearing zone around the colony on LB agar plates. Under the microscope, bacteria were characterized as rod-shaped and gram-positive. For catalase activity, all the strains were negative (no bubbles were observed). Based on the results of 16S rRNA sequencing, LB60 had high sequence identity (99.86\%) to Bacillus toyonensis strain BCT-7112, and LB68 had high sequence identity (99.93\%) to Bacillus toyonensis strain BCT-7112. A phylogenetic tree was constructed (Figure 1) using the 16S rRNA sequences, which demonstrated that, the selected strains belonged to Bacillus toyonensis. The BLAST result of the sequence were submitted to Genbank (accession numbers: MW287198 and MW287199 (LB60 and LB68 respectively). The new strains were named as Bacillus toyonensis SAU-19 (LB60) and Bacillus toyonensis SAU20 (LB68) respectively.

\section{Effects of Bacillus toyonensis SAU-19 and SAU-20 on Feed Intake and Weight of in Mice Fed High Fat Diet}

Based on our observations all the treatment groups appeared healthy and active. No deaths or signs of gastrointestinal upset like diarrhea occurred in the treatment groups (data not shown). In addition, Bacillus toyonensis SAU-19 and SAU-20 groups decreased the weight associated with high fat diet $(P<0.05)$ as compared to the HF group (Table 4) after the 35 days' experimental period. However, there was no difference between the Bacillus toyonensis (B. toyo.) groups and the LGG group $(P>0.05)$ in terms of weight gain. Weight gain in the $B$. toyo. SAU-19 group were also not significantly different from those in the B. toyo. SAU-20 group. Therefore, these results indicated that B. toyo. SAU-19 and B. toyo. SAU-20 could improve growth and immunity just as commercial probiotic 


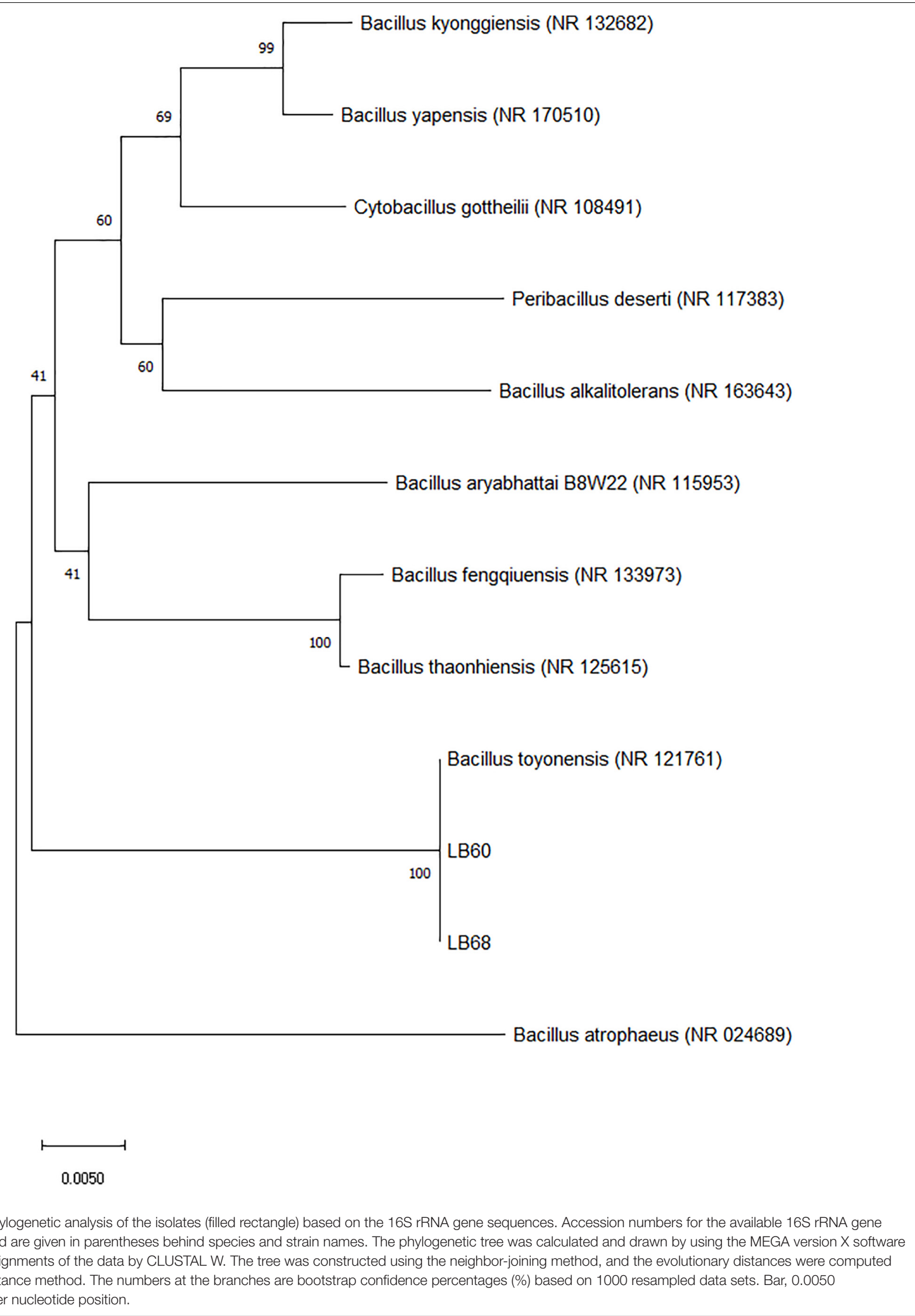


TABLE 4 | Effect of Bacillus toyonensis SAU-19 and SAU-20 on Average daily feed intake (g/d/mice) and Average weight (g/mice) of mice fed high fat diet.

\begin{tabular}{|c|c|c|c|c|c|}
\hline \multirow[b]{2}{*}{ Growth parameters } & \multicolumn{4}{|c|}{ Treatments } & \multirow[b]{2}{*}{$\mathrm{HF}+$ B. toyo. SAU-20 } \\
\hline & NC & HF & HF + LGG & $\mathrm{HF}+$ B. toyo. SAU-19 & \\
\hline Average Daily Feed intake ${ }^{1}$ (g/d/mice) & $5.00 \pm 0.002$ & $4.99 \pm 0.002$ & $4.99 \pm 0.02$ & $4.99 \pm 0.002$ & $5.00 \pm 0.002$ \\
\hline Initial Average body weight(g/mice) & $26.92 \pm 0.84$ & $26.49 \pm 0.17$ & $26.41 \pm 0.14$ & $26.97 \pm 0.24$ & $26.80 \pm 0.06$ \\
\hline Final Average body weight ${ }^{2}$ (g/mice) & $33.48 \pm 0.41^{b}$ & $37.82 \pm 1.13^{\mathrm{a}}$ & $34.83 \pm 0.28^{b}$ & $35.05 \pm 0.55^{b}$ & $35.16 \pm 1.39^{b}$ \\
\hline
\end{tabular}

The table is represented as means value \pm standard deviation $(S D)$. Columns with different superscripts ${ }^{a, b}$ are statistically different ( $\left.n=12, P<0.05\right)$.

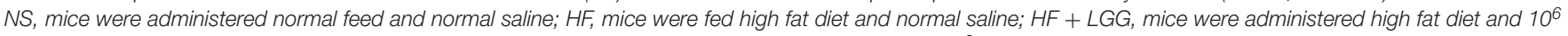

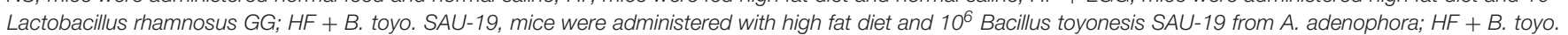
SAU-20, mice were administrated with high fat diet and $10^{6}$ Bacillus toyonensis SAU-20 from A. adenophora.

${ }^{1}$ Average daily feed intake $=$ Weight of feed given (g/day/mice)-Weight of feed remaining (g/day/mice).

${ }^{2}$ Average body weight $=$ Individual weights/total number of mice.

(LGG), hence can be used in treating obesity induced by high fat diets.

\section{Histopathological Results}

As shown in Figure $\mathbf{2 A}$, the structure of the small intestine of the control and treatment groups were complete and showed mild pathological changes compared to the HF group. The sub-mucosa, muscle layer and intestinal gland were arranged normally, and the morphology of the lamina propria and epithelial layer were complete. In addition, the mucosa and muscle layer in the treatment groups were clear compared to those in the HF group. The HF group showed moderate pathological changes characterized by intestinal structure damage, bleeding, and mild local inflammatory infiltration of the epithelial layers. Moreover, histological examination of ileum sections showed no significance difference in the cumulative injury scores of the treatment groups compared with the control group (Figure $\mathbf{2 B}, P<0.05$ ). The injury score for the HF group was higher that the control and the treatment groups $(P<0.05)$. Furthermore, there was no difference in the structure and injury scores among B. toyo. SAU-19, B. toyo. SAU20 and $L G G$ groups. These results indicated that, B. toyo. SAU-19 and $B$. toyo. SAU-20 could improve the structure of intestine from high fat diet induced injury.

\section{Effects of Bacillus toyonensis SAU-19 and SAU-20 on Villi Height and Crypt Depth of the Small Intestine in Mice Fed High Fat Diet}

The villi height (V) and crypt depth (C) of the ileum section of the small intestine of the high fat diet group (HF) was lower that the control whereas the $\mathrm{V}$ and $\mathrm{C}$ of the treatment groups were significantly higher than that of the HF group (Figure 3, $P<0.05)$. Moreover, there was no difference in villi height and crypt depth of the ileum section among the treatment groups $(P>0.05)$. Therefore, we stipulated that, B. toyo. SAU19 and B. toyo. SAU-20 protected or maintained the ileum structure from high fat diet destruction just as the commercial probiotic (LGG).

\section{Effects of Bacillus toyonensis SAU-19 and SAU-20 on Antioxidant Indexes and DAO Activity in Mice Fed High Fat Diet}

The results of this study showed that, all the treatment groups and control had reduced serum MDA levels compared to the HF group (Figure 4A, $P<0.05$ ). In contrast, SOD, CAT, and GSH levels in the treatment and control groups were significantly higher than those in the HF group (Figures 4B-D, $P<0.05$ ). However, we observed that the levels of MDA in the treatment groups were higher than the control (NC), whereas the levels of CAT and GSH in the treatment group lower than that of the control group $(P<0.05)$. There was no significance difference in SOD, CAT, and GSH levels among the LGG, B. toyo. SAU-19 and $B$. toyo. SAU-20 treatment groups $(P>0.05)$.

From the results, we also observed that, the levels of DAO in the treatment groups were lower than those in the HF group, however, the DAO levels of the treatment groups were significantly higher than the control group (Figure 4E, $p<0.05$ ). We also observed no difference among the NC, LGG, B. toyo. SAU-19 and B. toyo. SAU-20 groups $(P>0.05)$. These results indicate that the two isolated strains improved the barrier integrity of the intestine.

\section{Effects of Bacillus toyonensis SAU-19 and SAU-20 on sIgA Levels in the Small Intestine Contents of Mice Fed High Fat Diet}

For the sIgA levels in the small intestine contents, we observed that, the sIgA levels in the treatment groups were higher compared to the HF group (Figure 4F, $P<0.05$ ). We also observed that, the sIgA levels in the control (NC) and LGG group were higher than those in the B. toyo. SAU-20 $(P<0.05)$. However, no differences were observed among the NC, LGG and the B. toyo. SAU-19 groups $(P>0.05)$.

\section{Effects of Bacillus toyonensis SAU-19 and SAU-20 on Tight Junction Proteins in the Small Intestine Tissues of Mice Fed High Fat Diet}

The immune-histochemical analyses showed that, the occludin levels in treatment and control groups were increased as 



Small intestine

FIGURE 2 | Histopathological observation of in the small intestinal sections of mice fed Bacillus toyonensis SAU-19 and SAU-20 after 35 days. (A) Photograph of histopathological staining in treatment groups (Scale $=20 \mu \mathrm{m}$ ). (B) Histological inflammation damage scores of ileum section of the small intestine of mice. The values are presented as the mean \pm SD. Bars without the same letter differed significantly $(n=4, P<0.05)$. The HF group showed moderate pathological changes characterized by bleed, and moderate inflammation, however, these were mild in the treatment groups. Bleeds are indicated by red arrow and structural damage are indicated by black arrow. NC, mice were administered normal diet + normal saline; HF, mice were fed high fat diet + normal saline; HF + LGG, mice were administered high fat diet $+10^{6}$ Lactobacillus rhamnosus GG; HF + B. toyo. SAU- 19, mice were administered high fat diet + $10^{6}$ Bacillus toyonensis SAU-19 from A. adenophora; HF + B. toyo. SAU-20, mice were administrated with high fat diet $+10^{6}$ Bacillus toyonensis SAU-20 from A. adenophora.

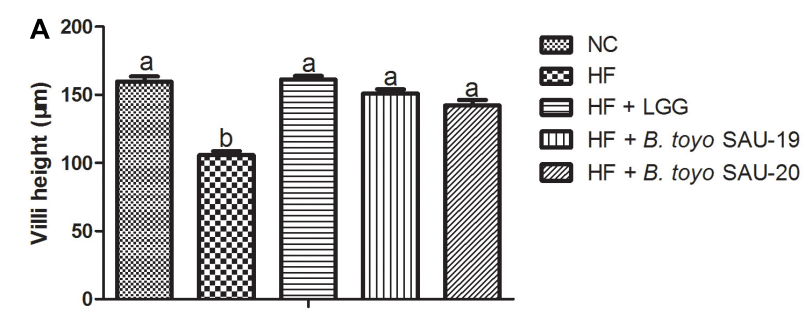

ileum



ileum

FIGURE 3 | Effects of Bacillus toyonensis SAU-19 and SAU-20 on the villi height and crypt depth of small intestine in mice fed high fat diet. (A) Villi height of ileum in mice. (B) Crypt depth of ileum in mice. The values are presented as the mean \pm SD. Bars without the same letter differed significantly $(n=4, P<0.05)$. NC, mice were administered normal diet + normal saline; HF, mice were fed high fat diet + normal saline; HF + LGG, mice were administered high fat diet + $10^{6}$ Lactobacillus rhamnosus GG; HF + B. toyo. SAU-19, mice were administered high fat diet $+10^{6}$ Bacillus toyonensis SAU-19 from A. adenophora; HF $+B$. toyo. SAU-20, mice were administrated with high fat diet $+10^{6}$ Bacillus toyonensis SAU-20 from A. adenophora.

compared to the HF group (Figures 5A,B, $P<0.05$ ). Furthermore, we observed that, the level of occuldin in the LGG and B. toyo. SAU-19 groups was significantly higher than that of the B. toyo. SAU- 20 groups $(P<0.05)$, but no difference was observed between the LGG and the B. toyo. SAU-19 groups. Furthermore, the results of the relative mRNA expression of tight junction proteins (TJP) showed that, the levels of both TJPs (occludin and Z0-1) were significantly higher in the treatment groups as compared to the HF group (Figures 5C,D, $P<0.05$ ). However, the LGG and B. toyo. SAU-19 group had an elevated expression levels of occludin and ZO-1 compared to the B. toyo.
SAU-20 group $(P<0.05)$. No difference existed between the LGG and the B. toyo. SAU-19 groups.

\section{Effects of Bacillus toyonensis SAU-19 and SAU-20 on Relative mRNA Expressions Pro- and Anti-inflammation Related Cytokines in the Small Intestine Tissues of Mice Fed High Fat Diet}

The results of the relative mRNA expression of inflammation related cytokines showed that, the treatment groups decreased 

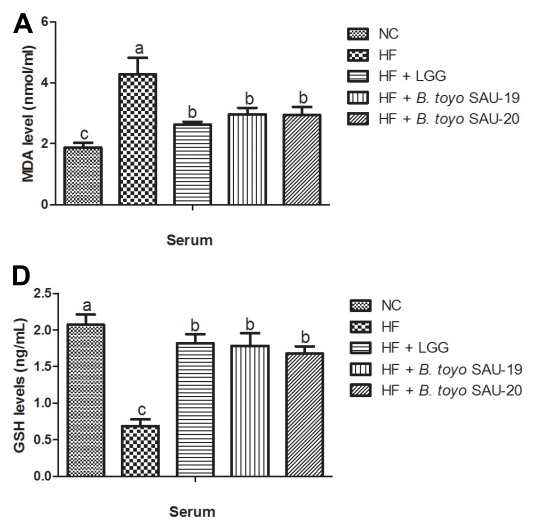



Serum



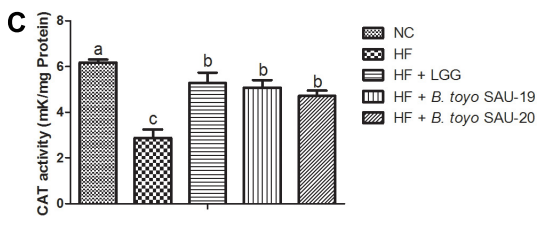

Serum

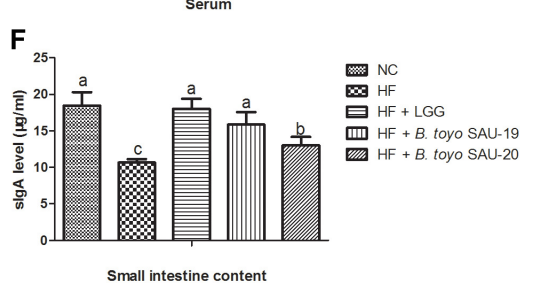

FIGURE 4 | Effect of Bacillus toyonensis SAU-19 and SAU-20 on antioxidant indexes, DAO and slgA activity in mice fed high fat diet (A) levels of MDA enzyme in serum, (B) levels of SOD enzyme in serum, (C) levels of CAT enzyme in serum (D) levels of GSH enzyme in serum (E) DAO concentrations in the serum, (F) slgA levels in small intestine contents. Bars represent means \pm standard deviation (SD). The values are presented as the mean \pm SD. Bars without the same letter differed significantly $(n=6, P<0.05)$. NC, mice were administered normal diet + normal saline; HF, mice were fed high fat diet + normal saline; HF + LGG, mice were administered high fat diet $+10^{6}$ Lactobacillus rhamnosus GG; HF $+B$. toyo. SAU-19, mice were administered high fat diet $+10^{6}$ Bacillus toyonensis SAU-19 from A. adenophora; HF + B. toyo. SAU-20, mice were administrated with high fat diet $+10^{6}$ Bacillus toyonensis SAU-20 from A. adenophora.

the levels of pro-inflammatory cytokines (IL-1 $\beta$, TFN- $\alpha$, IL-2) and increased the level of the anti-inflammatory cytokines (IL-4 and IL-10) in small intestine tissues as compared to the HF group (Figures 6A-E, $P<0.05$ ). Moreover, there was no difference in both pro- and anti-inflammatory cytokines among the B. toyo. SAU-19, B. toyo. SAU-20 and LGG groups $(P>0.05)$.

\section{Effects of Bacillus toyonensis SAU-19 and SAU-20 on Pathogenic (Escherichia coli, Salmonella, and Staphylococcus aureus) and Beneficial (Lactobacillus spp.) Bacteria Enumeration in Small Intestine and Cecum Contents in Mice Fed High Fat Diet}

The results of the microbial count of the small intestine and cecum contents are shown in Figures 7, 8. These results showed that, the E. coli, Salmonella spp., and Staphylococcus aureus (S. aureus) counts (population) in the small intestine contents was significantly lower in the Control, LGG, B. toyo. SAU-19 and B. toyo. SAU-20 treatment groups as compared to the $\mathrm{HF}$ group (Figures 7A-C, $P<0.05$ ). Moreover, both $B$. toyo. SAU19 and $B$. toyo. SAU-20 treatment groups increased the number of Lactobacillus spp bacteria in the small intestine contents as compared to the HF (Figure 7D, $P<0.05$ ).

Similarly, the count of E. coli, salmonella, and S. aureus in the cecum content were significantly lower in the Control, B. toyo. SAU-19 and B. toyo. SAU-20 treatment groups compared to the HF group (Figures $\mathbf{8 A}-\mathrm{C}, P<0.05$ ). Finally, the B. toyo. SAU-19 and $B$. toyo. SAU-20 treatment groups increased the number of Lactobacillus spp. bacteria in the cecum as compared to the HF group (Figure 8D, $P<0.05$ ). Moreover, there was no difference in bacteria enumeration between B. toyo. SAU-19 and B. toyo. SAU-20. We further observed that the counts of Salmonella and $S$. aureus in the small intestine content of the LGG group was lower compared to the B. toyo. SAU-19 and B. toyo. SAU-20 $(P<0.05)$. In addition, the Lactobacillus spp. count in the small intestine of the LGG group was higher than that of $B$. toyo. SAU-19 and B. toyo. SAU-20 $(P<0.05)$.

\section{DISCUSSION}

Bacillus toyonensis is a naturally occurring, non-toxigenic and non-pathogenic strain of $B$. cereus, and which does not cause any adverse effects in animals such as rabbits, pigs, chickens, turkeys and cattle (Jimenez et al., 2013; EFSA, 2014). Many studies have revealed probiotic activities of the bacteria (Taras et al., 2005). Bacillus toyonensis have been reported to increase weight gain, improved feed conversion ratios, reduce postweaning diarrhea and lower mortality rates of piglets (Baum et al., 2002). The results of our 16S rRNA and the phylogenetic tree demonstrated that, the selected probiotic endophyte strains were similar to Bacillus toyonensis and the morphological analysis results showed that, the strains had similar morphology as the general characteristics of Bacillus spp. used as probiotics (Özüsağlam and Aksaray, 2010), however, differed in some colony morphological characteristics like margins, elevation and colony size as well as sugar fermentation. Therefore, we hypothesized that Bacillus toyonensis SAU-19 (LB60) and SAU-20 (LB68) may possess probiotic properties. Both tested endophytes successfully fermented glucose and sucrose except for D-sorbitol which was fermented only by $\mathrm{LB} 60$, were tolerant to $\mathrm{NaCl}$, low $\mathrm{pH}$ and bile salt. This results were similar to the works of Nithya and Halami (2013) and Mok et al. (2020).

Moreover, the antibiotic resistance and antimicrobial activities of probiotic strains are essential to prevent or reduce infections caused by pathogenic bacteria. In this study, it was observed that, both Bacillus toyonensis (LB60 and LB68) were resistant to ampicillin and erythromycin, however, they were susceptible to streptomycin, tetracycline, and sultamicillin according to 

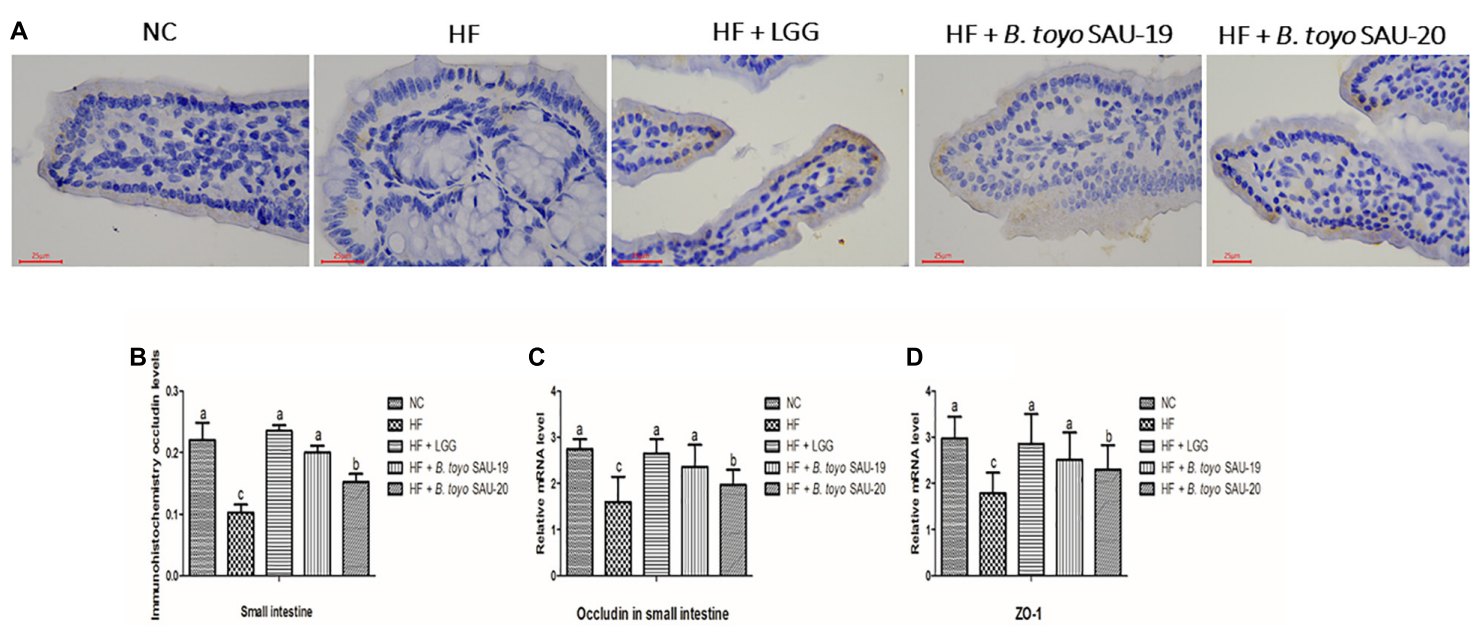

FIGURE 5 | Effects of Bacillus toyonensis SAU-19 and SAU-20 on tight junction proteins of the small intestine tissue in mice fed high fat diet.

(A) Immunohistochemistry staining photographs of intestinal sections (yellow area represents occludin). (B) Immunohistochemical analyses of occludin levels in small intestine of mice in each group Bars represent means \pm standard deviation (SD), the values are presented as the mean \pm SD. Bars without the same letter differed significantly $(n=4, P<0.05)$. (C) mRNA expression of occludin in small intestine tissues (D) mRNA expression of ZO-1 in small intestine tissues. Bars represent means \pm standard deviation (SD) for eight mice per treatment. The values are presented as the mean $\pm \mathrm{SD}$. Bars without the same letter differed significantly $(p<0.05)$. NC, mice were administered normal diet + normal saline; HF, mice were fed high fat diet + normal saline; HF + LGG, mice were administered high fat diet $+10^{6}$ Lactobacillus rhamnosus GG; HF + B. toyo. SAU-19, mice were administered high fat diet $+10^{6}$ Bacillus toyonensis SAU-19 from A. adenophora; $\mathrm{HF}+$ B. toyo. SAU-20, mice were administrated with high fat diet $+10^{6}$ Bacillus toyonensis SAU-20 from A. adenophora.
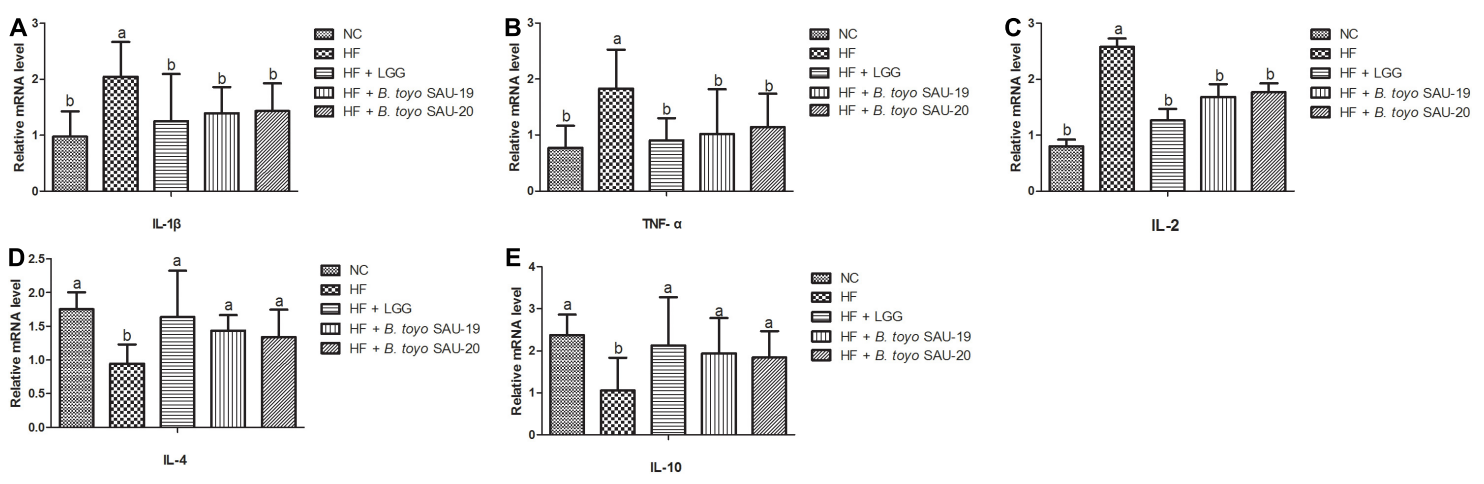

FIGURE 6 | Effects of Bacillus toyonensis SAU-19 and SAU-20 on relative mRNA expressions of pro- and anti-inflammation related cytokines in the small intestine tissue of mice fed high fat diet (A-C) mRNA expressions of pro-inflammatory cytokines (D,E) mRNA expressions of anti-inflammatory cytokines. Bars represent means \pm standard deviation (SD). The values are presented as the mean \pm SD. Bars without the same letter differed significantly $(n=6, P<0.05)$. NC, mice were administered normal diet + normal saline; HF, mice were fed high fat diet + normal saline; HF + LGG, mice were administered high fat diet + 106 Lactobacillus rhamnosus GG; HF + B. toyo. SAU-19, mice were administered high fat diet $+10^{6}$ Bacillus toyonensis SAU-19 from A. adenophora; HF $+B$. toyo. SAU-20, mice were administrated with high fat diet $+10^{6}$ Bacillus toyonensis SAU-20 from A. adenophora.

the method described by Talib et al. (2019). This results were consistent with the study of Glenwright et al. (2017) and Fiedler et al. (2019). Furthermore, both Bacillus toyonensis (LB60 and LB68) had no inhibitory effect on tested pathogenic bacteria, hence, we concluded that, the endophytic bacteria did not produce antimicrobial substances. This finding were similar to the study of Agamennone et al. (2019) and Rojas-Solis et al. (2020).

High fat diets has been reported to increase weight gain, affect immune organ weight and indices (Jin et al., 2017; Li et al., 2020; Yoshizaki et al., 2020). Probiotics reduce excessive weight increase in animals fed high fat diet (Kong et al., 2019; Chen Y. T. et al., 2021). From the results of the in vivo experiments, we observed that, Bacillus toyonensis SAU-19 and SAU-20 could reduce weight gain in mice compared to the HF group. Similar results were obtained from a previous study by Kantas et al. (2015), and Cai et al. (2020) which reported that, probiotics could reduce the weights associated with high fat diet in animals.

Numerous studies have reported on the association of high fat diet to the distortion of the gut microbiota and pathological changes in the intestine (Netto Candido et al., 2018). This may be as a results of increasing harmful bacteria and 

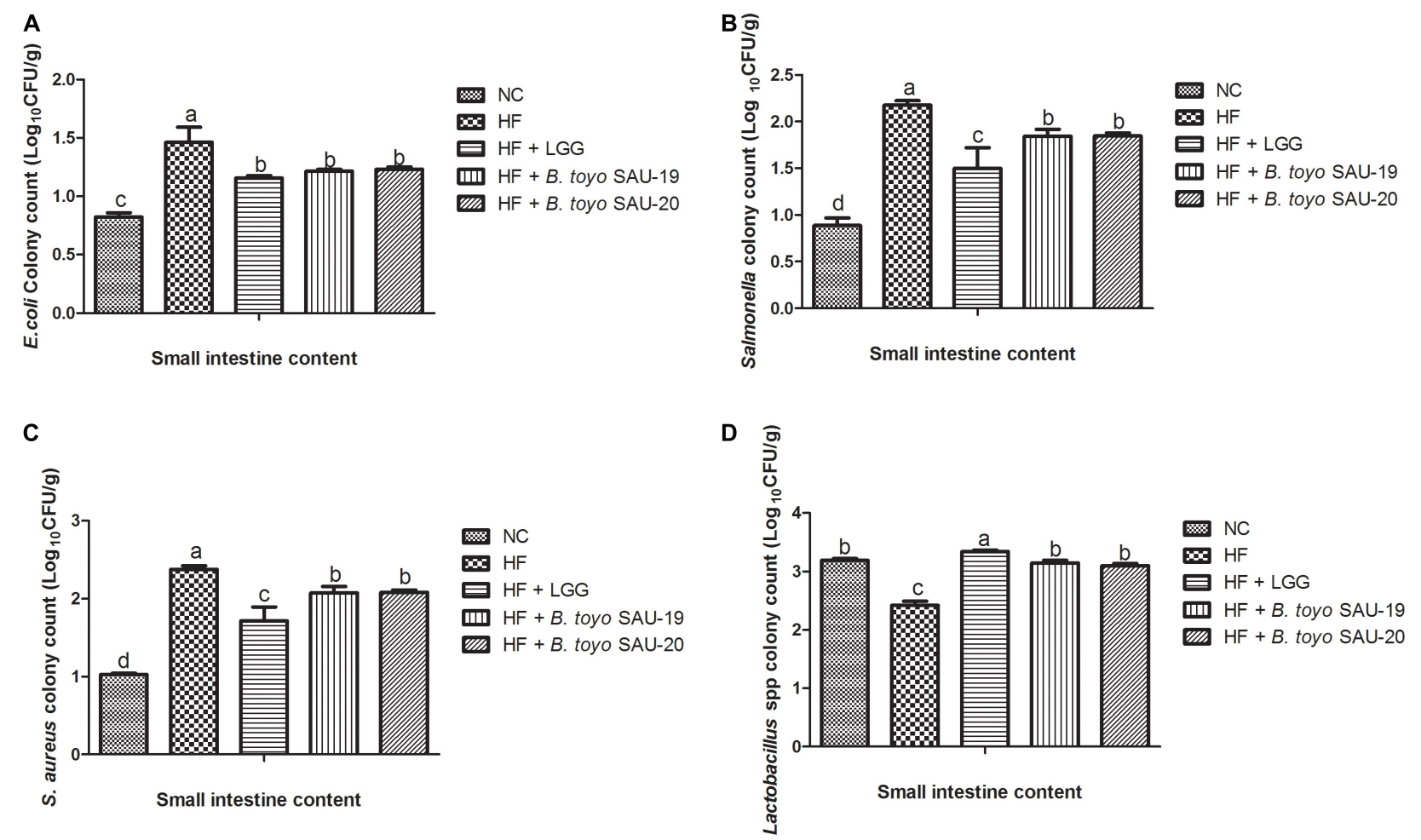

FIGURE 7 | Effects of Bacillus toyonensis SAU-19 and SAU-20 on bacteria enumeration in small intestine contents of mice fed high fat diet (A) colony count of Escherichia coli, (B) colony count of Salmonella spp., (C) colony count of Staphylococcus aureus, (D) colony count of Lactobacillus spp. The values are presented as the mean $\pm \mathrm{SD}$. Bars without the same letter differed significantly $(n=6, P<0.05)$ the same. NC, mice were administered normal diet + normal saline; HF, mice were fed high fat diet + normal saline; HF + LGG, mice were administered high fat diet + 10 6 Lactobacillus rhamnosus GG; HF + B. toyo. SAU-19, mice were administered high fat diet $+10^{6}$ Bacillus toyonensis SAU-19 from A. adenophora; HF + B. toyo. SAU-20, mice were administrated with high fat diet $+10^{6}$ Bacillus toyonensis SAU-20 from A. adenophora.

viruses which invade tissues by producing lethal toxins and metabolites which may cause damages in the GIT (Abaidullah et al., 2019). The pathological results obtained in this study showed that administering Bacillus toyonensis SAU-19 and SAU-20 did not cause any severe pathological changes in the small intestine tissues as compared to the HF group that showed a moderate pathological changes. Complications such as bleeding, and moderate inflammation were observed in the small intestines of mice in the HF group, however this was mild in the treatment groups. This was consistent with previous reports by Ringø et al. (2007) who stated that, probiotics could reverse the intestinal pathological damage caused by pathogenic bacteria.

Furthermore, villus height (V), crypt depth (C), and V:C ratio are major morphological indicators that are used to determine the general health and functions of the small intestine (Feng et al., 2016; Cui et al., 2021). Villi are important structures found in the small intestine and are mainly involved in the absorption of nutrients (Fuller, 2004). The higher the villi height and the deeper the crypt depth the higher the surface area for nutrient absorption in the small intestine (Chwen et al., 2013). In this study, it was observed that, the ileum section of the small intestine in the treatment groups had significantly higher villi heights and crypt depth as compared to the high fat diet (HF) group, indicating that, Bacillus toyonensis SAU-19 and SAU-20 could improve the villi structure thereby increasing its height and crypt depth. This result was consistent with a previous study by Jayaraman et al. (2013) and Wang et al. (2019) who reported that, probiotics could improve villi length and crypt depth in animals.

The intestinal barrier is a complex multilayer system, consisting of an external anatomical barrier and an inner functional immunological barrier (Zheng et al., 2019). Chemical markers (such as DAO and D-lactic acid) are important indicators of intestinal permeability. When the intestinal barrier function is damaged, these chemical makers are released into the blood and these are employed as indicators for intestinal integrity (Chen F. et al., 2021). Our results showed that, all the treatment groups could reduce the levels of DAO in mouse serum, however, only LGG and Bacillus toyonensis SAU-19 showed a significant reduction compared to the $\mathrm{HF}$ group. This is similar to other studies that reported that, probiotics reduce the level of DAO in the serum (Ding et al., 2019).

Oxidative stress is a harmful physiological reaction, which can lead to excessive re-active oxygen species (ROS) generation (Pizzino et al., 2017). Various studies have reported that; oxidative stress reduces growth performance in animals (Lv et al., 2012). MDA is an oxidative stress indicator whereas SOD, CAT and GSH-PX are main antioxidant enzymes in mammals (Wagner 




et al., 1994; Ighodaro and Akinloye, 2018). High fat diet has been linked with oxidative stress ( $\mathrm{Li}$ et al., 2019; Tan and Norhaizan, 2019). Numerous studies have also reported that supplementation of exogenous probiotics has a beneficial effect on preventing oxidative stress. Our results showed that Bacillus toyonensis SAU-19 and SAU-20 reduced the MDA levels and increased the SOD, CAT, and GSH activity in the serum. This result is consistent with a previous study by Yau et al. (2020) which reported that, Lactobacillus rhamnosus GG could improve the antioxidant capacity of mice fed high fat diet.

Secretory immunoglobulin A (sIgA) is a predominant immunoglobulin in the mucosal system and is critical for protecting mucosal surfaces against toxins, viruses, and enteropathogens (Xin et al., 2020). It neutralizes or prevent pathogens from binding to the mucosal surface of the intestine (Lindner et al., 2012), thereby protecting the intestine. sIgA also plays a role in the preservation of mucosal homeostasis, which determines the intestinal microbiota composition and enhances the development of systemic immunity (Lammers et al., 2010). Furthermore, sIgA suppresses the release of pro-inflammation indicators that initiate intestinal diseases by coating a portion of commensal intestinal bacteria in humans and animals (Van et al., 2004; Palm et al., 2014; Xin et al., 2020).
Our results showed that both Bacillus toyonensis SAU-19 and SAU-20 increased sIgA levels in the small intestine contents. This result indicated that, these two strains improved intestinal health. The results of this study are consistent with a previous study by Kabeerdoss et al. (2011) which reported that, probiotic interventions could increase the fecal IgA production in healthy children, preterm infants, and piglets.

Intestinal TJP, such as claudin, ZO, and occludin, have been reported to be directly associated with the normal function of the intestinal mucosa barrier (Wu et al., 2018). The leaking gut is often associated with reduced expression or internalization of TJPs such as occludin and ZO (Buschmann et al., 2013). High-fat diet (HFD)-feeding increases intestinal permeability by impairing tight junction (TJ) protein function, which may explain the associated pathologies (Chen K. et al., 2020). Our results showed that, both Bacillus toyonensis SAU-19 and SAU20 increased the expression levels of TJ proteins (Occludin and $\mathrm{ZO}-1)$ in the small intestine of high fat diet fed-mice thereby conferring a beneficial effect on mucosal barrier function. This is in agreement with a previous study by Gong et al. (2016) which reported that, Bacillus subtilis administration could elevate the expression of TJ proteins (claudin-1, occludin, JAM-A, and $\mathrm{ZO}-1$ ) in mice. 
Furthermore, the abnormal expression of TJP is closely associated with the inflammatory responses of the intestine (Xu et al., 2016). Cytokines, as endogenous mediators of the immune system that control the occurrence of inflammatory reaction (Jiang et al., 2019). When there is acute toxicity (such as toxin metabolism), the levels of IL-6, IL-2, tumor necrosis factor and other inflammatory factors increase (Zhang et al., 2018). The intestinal barrier does not only protect the deeper layers of the intestinal wall, but also tightly regulates the passage of pro-inflammatory molecules, microorganisms, toxins, and antigens (Farré et al., 2020). High expression of proinflammation cytokines such as IL- $1 \beta$ and TNF- $\alpha$, encourage intestinal epithelial cells damage via autocrine/paracrine action (Van Kaer and Olivares-Villagomez, 2018). High fat diet causes inflammation characterized by elevated levels of proinflammation cytokines (van der Heijden et al., 2015; Dutheil et al., 2016). Our results showed that, Bacillus toyonensis SAU19 and SAU-20 could reduce the expression of pro-inflammatory cytokine and increase the expressions of the anti-inflammation cytokine in small intestine compared to the high fat diet group, thereby improving immunity. This study is in agreement with a previous study by Roos et al. (2018).

The gut microbiota is altered by feeding high fat diet (Bibbò et al., 2016; Guo et al., 2017). Exogenous probiotics improve growth performance by altering gut microbiota (Xin et al., 2020). Our current study showed that Bacillus toyonensis SAU-19 and SAU-20 isolated from $A$. adenophora exert beneficial effects on intestinal flora. Specifically, Bacillus toyonensis SAU-19 and SAU-20 markedly enhanced Lactobacillus spp. populations and reduced Enterobacteriaceae populations (E. coli, Salmonella, and Staphylococcus aureus) compared to the high fat diet group. Many studies have reported that, Bacillus toyonensis could inhibit the activities of Salmonella enteritidis and E. coli in the gut of pigs (Simon, 2010). Therefore, it was speculated that, Bacillus toyonensis SAU-19 and SAU-20 played a role in reducing the harmful bacteria and increasing beneficial bacteria thus, balancing the gut microbiota. However, the mechanism for achieving this activity was not from the production of antimicrobial substances and this was confirmed by the antimicrobial test in this study. Even though a study by GonzalezOrtiz et al. (2016) reported that, Bacillus toyonensis effectively reduced $E$. coli activity in the gut mainly by inhibiting the quorum sensing of the pathogenic bacteria there is still the need for an in-depth study to reveal the main molecular mechanism of these strains gut modulation activities.

In summary of the results, this study reported on the cross talk between gut microbiota and probiotics. We observed changes in the gut microbiota composition in the high fat diet fedmice characterized by an increase in the pathogenic bacteria population. In addition, we also observed that the intestinal structure and barrier was damaged in the high fat diet fed-mice. However, we did not observe these changes in the normal mice. Therefore, we concluded that, the impairment of the gut structure and integrity was as a result of dysbiosis of the gut microbiota. Furthermore, the administration of SAU-19 and SAU-20 did not cause any obvious damage to the intestine or change the gut microbiota composition compared to the normal mice, hence we concluded that these two strains protected or prevented the intestine from damages associated with gut dysbiosis in mice fed high fat diet.

Even though this study has revealed the probiotic potential of the B. toyonensis SAU-19 and SAU-20, this study did not investigate the main mode of action of these bacteria strains in reducing the population of the pathogenic bacteria in the gut. In addition, we only quantified the limited small intestine and cecum microbial contents using the plate count method. Moreover, this study also lacked the correlation analysis of the gut microbiota and their metabolites function in improving the gut health. Thus, there is the need for further research to ascertain the exact molecular mechanisms of these two strains in maintain the gut structure and integrity as well as performing microbial diversity analysis based on next-generation sequencing to clearly reveal the complete changes and correlation of the gut microbiota and their metabolites as a results of Bacillus toyonensis SAU-19 and SAU-20 administration.

\section{CONCLUSION}

We performed a preliminary study and isolated and identified two potential probiotic bacteria endophytes (Bacillus toyonensis SAU-19 and SAU- 20) from A. adenophora. Both Bacillus toyonensis strains prevented the excessive increase in body weights associated with high fat diet feeding, improved the antioxidant status, alleviated structure and integrity damage of the small intestine and reduced the levels of pathogenic bacteria (E. coli, Salmonella, and Staphylococcus aureus) without causing any health complications in mice fed high fat diet. Therefore, Bacillus toyonensis SAU-19 and SAU-20 may be used as future probiotics in humans and animals. However, further studies are still needed to clarify the detailed mechanisms of action by validating the efficacy of B. toyonensis SAU-19 and SAU-20 through human clinical trials.

\section{DATA AVAILABILITY STATEMENT}

Publicly available datasets were analyzed in this study. This data can be found here: MW287198 and MW287199.

\section{ETHICS STATEMENT}

The animal study was reviewed and approved by Institutional Animal Care and Use Committee of Sichuan Agricultural University, Sichuan, China, under the permit number DKYB2019603005.

\section{AUTHOR CONTRIBUTIONS}

SO, JW, YC, and LX: conceptualization, methodology, and software. SO, LX, JW, SW, YR, PG, and MZ: data collection, writing, and original draft preparation. SO, YC, ZR, and $\mathrm{YH}$ : validation and investigation. JW, $\mathrm{ZR}$, and $\mathrm{YH}$ : funding and 
supervision. All authors read and agreed to the published version of the manuscript.

\section{FUNDING}

This research was supported by the Science and Technology Support Program (Grant No. 2020YFS0337).

\section{REFERENCES}

Abaidullah, M., Peng, S., Kamran, M., Song, X., and Yin, Z. (2019). Current findings on gut microbiota mediated immune modulation against viral diseases in chicken. Viruses 11:681. doi: 10.3390/v11080681

Agamennone, V., Straalen, J., Brouwer, A., Boer, T. E., Hensbergen, P. J., Zaagman, N., et al. (2019). Genome annotation and antimicrobial properties of Bacillus toyonensis VU-DES13, isolated from the Folsomia candida gut. Entomol. Exp. Appl. 167, 269-285.

Ahmad, M. S., and Zargar, M. Y. (2017). Characterization of potassium solubilizing bacteria (KSB) in rhizospheric soils of Apple (Malus domestica Borkh) in Temperate Kashmir. J. Appl. Life Sci. Int. 15, 1-7. doi: 10.9734/jalsi/2017/3 6848

Alfonzo, A., Miceli, C., Nasca, A., Franciosi, E., Ventimiglia, G., Di Gerlando, R., et al. (2016). Monitoring of wheat lactic acid bacteria from the field until the first step of dough fermentation. Food Microbiol. 62, 256-269. doi: 10.1016/j. fm.2016.10.014

Baum, B., Liebler-Tenorio, E. M., Enss, M. L., Pohlenz, J. F., and Breves, G. (2002). Saccharomyces boulardii and Bacillus cereus var. toyoi influence the morphology and the mucins of the intestine of pigs. Zeitschrift Fur Gastroenterol. 40, 277-284. doi: 10.1055/s-2002-30116

Bhore, S. J., Ravichantar, N., and Loh, C. Y. (2010). Screening of endophytic bacteria isolated from leaves of Sambung nyawa [Gynura procumbens (Lour.) Merr.] for cytokinin-like compounds. Bioinformation 5, 191-197. doi: 10.6026/ 97320630005191

Bibbò, S., Ianiro, G., Giorgio, V., Scaldaferri, F., Masucci, L., Gasbarrini, A., et al. (2016). The role of diet on gut microbiota composition. Eur. Rev. Med. Pharmacol. Sci. 20, 4742-4749.

Buschmann, M. M., Shen, L., Rajapakse, H., Raleigh, D. R., Wang, Y., Wang, Y., et al. (2013). Occludin OCEL-domain interactions are required for maintenance and regulation of the tight junction barrier to macromolecular flux. Mol. Biol. Cell 24, 3056-3068. doi: 10.1091/mbc.E12-09-0688

Cai, H., Wen, Z., Li, X., Meng, K., and Yang, P. (2020). Lactobacillus plantarum FRT10 alleviated high-fat diet-induced obesity in mice through regulating the PPAR $\alpha$ signal pathway and gut microbiota. Appl. Microbiol. Biotechnol. 104, 5959-5972. doi: 10.1007/s00253-020-10620-0

Cani, P. D., Amar, J., Iglesias, M. A., Poggi, M., Knauf, C., Bastelica, D., et al. (2007). Metabolic endotoxemia initiates obesity and insulin resistance. Diabetes 56, 1761-1772.

Chakraborty, A., and Bhowal, J. (2015). Isolation, identification and analysis of probiotic properties of Lactobacillus Spp. from selected regional dairy product. Int. J. Curr. Microbiol. Appl. Sci. 4, 621-628.

Chen, F., Chen, J., Chen, Q., Yang, L., Yin, J., Li, Y., et al. (2021). Lactobacillus delbrueckii protected intestinal integrity, alleviated intestinal oxidative damage, and activated toll-like receptor-bruton's tyrosine kinase-nuclear factor erythroid 2-Related factor 2 pathway in weaned piglets challenged with lipopolysaccharide. Antioxidants 10:468. doi: 10.3390/antiox10030468

Chen, K., Zhao, H., Shu, L., Xing, H., Wang, C., Lu, C., et al. (2020). Effect of resveratrol on intestinal tight junction proteins and the gut microbiome in high-fat diet-fed insulin resistant mice. Int. J. Food Sci. Nutr. 71, 965-978. doi: 10.1080/09637486.2020.1754351

Chen, L., Zhou, J., Zeng, T., Miao, Y., Mei, L., Yao, G., et al. (2020). Quantifying the sharing of foliar fungal pathogens by the invasive plant Ageratina adenophora and its neighbours. New Phytol. 227, 1493-1504. doi: 10.1111/nph.16624

Chen, Y. T., Hsu, A. H., Chiou, S. Y., Lin, Y. C., and Lin, J. S. (2021). ABKefir reduced body weight and ameliorated inflammation in adipose tissue of

\section{ACKNOWLEDGMENTS}

I would like to thank all authors for their hard work in making this paper publishable. I also extend my sincere gratitude to the teaching staff of the College of Veterinary Medicine, Sichuan Agricultural University, Chengdu, for their guidance and criticisms in writing this manuscript.

obese mice fed a high-fat diet, but not a high-sucrose diet. Nutrients 13:2182. doi: $10.3390 /$ nu13072182

Chwen, L., Foo, H., Thanh, N., and Choe, D. (2013). Growth performance, plasma fatty acids, villous height and crypt depth of preweaning piglets fed with medium chain triacylglycerol. Asian Austral. J. Anim. Sci. 26, 700-704. doi: 10.5713/ajas.2012.12561

Cui, Y., Okyere, S. K., Gao, P., Wen, J., Cao, S., Wang, Y., et al. (2021). Ageratina adenophora disrupts the intestinal structure and immune barrier integrity in rats. Toxins (Basel) 13:651. doi: 10.3390/toxins1309 0651

Delgado, T. C., Pinheiro, D., Caldeira, M., Castro, M. M., Geraldes, C. F., LópezLarrubia, P., et al. (2009). Sources of hepatic triglyceride accumulation during high-fat feeding in the healthy rat. NMR Biomed. 22, 310-317. doi: 10.1002/ nbm.1327

Ding, S., Wang, Y., Yan, W., Li, A., Jiang, H., and Fang, J. (2019). Effects of Lactobacillus plantarum 15-1 and fructooligosaccharides on the response of broilers to pathogenic Escherichia coli O78 challenge. PLoS One 14:e0212079. doi: 10.1371/journal.pone.0212079

Dutheil, S., Ota, K. T., Wohleb, E. S., Rasmussen, K., and Duman, R. S. (2016). High-fat diet induced anxiety and anhedonia: impact on brain homeostasis and inflammation. Neuropsychopharmacology 41, 1874-1887. doi: 10.1038/npp. 2015.357

EFSA (2014). FEEDAP panel (EFSA panel on additives and products or substances used in animal feed) scientific opinion on the safety and efficacy of Toyocerin (Bacillus toyonensis) as a feed additive for rabbits for fattening, chickens for fattening, weaned piglets, pigs for fattening, sows for reproduction, cattle for fattening, and calve s for rearing. EFSA J. 12:17.

Fan, L., Qi, Y., Qu, S., Chen, X., Li, A., Hendi, M., et al. (2021). B. adolescentis ameliorates chronic colitis by regulating Treg/Th2 response and gut microbiota remodeling. Gut Microbes 13, 1-17. doi: 10.1080/19490976.2020.1826746

FAO/WHO (2002). Guidelines for the Evaluation of Probiotics in Food. Geneva: World Health Organization (WHO) Food and Agriculture Organization (FAO).

Farré, R., Fiorani, M., Abdu Rahiman, S., and Matteoli, G. (2020). Intestinal permeability, inflammation and the role of nutrients. Nutrients 12:1185. doi: 10.3390/nu12041185

Feng, Z., Li, T., Wu, C., Tao, L., Blachier, F., and Yin, Y. (2016). Monosodium L-glutamate and dietary fat exert opposite effects on the proximal and distal intestinal health in growing pigs. Appl. Physiol. Nutr. Metab. 40, 353-363. doi: 10.1139/apnm-2014-0434

Fiedler, G., Schneider, C., Igbinosa, E. O., Kabisch, J., Brinks, E., Becker, B., et al. (2019). Antibiotics resistance and toxin profiles of Bacillus cereus-group isolates from fresh vegetables from German retail markets. BMC Microbiol. 19:250. doi: 10.1186/s12866-019-1632-2

Fisher, P. J., Petrini, O., and Scott, H. M. L. (1992). The distribution of some fungal and bacterial endophytes in maize (Zea mays L.). New Phytol. 122, 299-305. doi: 10.1111/j.1469-8137.1992.tb04234.x

Fu, J., Hu, L., Shi, Z., Sun, W., Yue, D., Wang, Y., et al. (2019). Two metabolites isolated from endophytic fungus Coniochaeta sp. F-8 in Ageratina adenophora exhibit antioxidative activity and cytotoxicity. Nat. Prod. Res. 1-9. doi: 10.1080/ 14786419.2019.1675060

Fuller, M. (2004). The Encyclopedia of Farm Animal Nutrition. Wallingford: CAB International, 244-246.

Glenwright, H., Pohl, S., Navarro, F., Miro, E., Jiménez, G., Blanch, A. R., et al. (2017). The identification of intrinsic chloramphenicol and tetracycline resistance genes in members of the Bacillus cereus group (sensu lato). Front. Microbiol. 7:2122. doi: 10.3389/fmicb.2016.02122 
Gong, Y., Li, H., and Li, Y. (2016). Effects of Bacillus subtilis on epithelial tight junctions of mice with inflammatory bowel disease. J. Interferon. Cytokine Res. 36, 75-85. doi: 10.1089/jir.2015.0030

Gonzalez-Ortiz, G., Sola-Oriol, D., Cerda-Cuellar, M., Castello, A., Castillo, M., and Mart $\iota n$-Orue, S. M. (2016). Study of the ability of Bacillus toyonensis to interfere with the quorum-sensing systems of enterotoxigenic Escherichia coli K88 in the pig gut. J. Anim. Sci. 94, 70-74.

Guo, X., Li, J., Tang, R., Zhang, G., Zeng, H., Wood, R. J., et al. (2017). High fat diet alters gut microbiota and the expression of paneth cell-antimicrobial peptides preceding changes of circulating inflammatory cytokines. Mediators Inflamm. 2017:9474896. doi: 10.1155/2017/9474896

He, Y., Mo, Q., Luo, B., Qiao, Y., Xu, R., Zuo, Z., et al. (2016). Induction of apoptosis and autophagy via mitochondria- and PI3K/Akt/mTOR-mediated pathways by E. adenophorum in hepatocytes of saanen goat. Oncotarget 7, 54537-54548. doi: 10.18632/oncotarget.10402

Ighodaro, O. M., and Akinloye, O. A. (2018). First line defence antioxidantssuperoxide dismutase (SOD), catalase (CAT) and glutathione peroxidase (GPX): Their fundamental role in the entire antioxidant defence grid. Alexandria J. Med. 45, 286-293.

Jang, Y. J., Kim, W. K., Han, D. H., Lee, K., and Ko, G. (2019). Lactobacillus fermentum species ameliorate dextran sulfate sodium-induced colitis by regulating the immune response and altering gut microbiota. Gut Microbes 10, 696-711. doi: 10.1080/19490976.2019.1589281

Jayaraman, S., Thangavel, G., Kurian, H., Mani, R., Mukkalil, R., and Chirakkal, H. (2013). Bacillus subtilis PB6 improves intestinal health of broiler chickens challenged with Clostridium perfringens-induced necrotic enteritis. Poult. Sci. 92, 370-374. doi: 10.3382/ps.2012-02528

Jiang, N., Li, Y., Shu, T., and Wang, J. (2019). Cytokines and inflammation in adipogenesis: an updated review. Front. Med. 13:314-329. doi: 10.1007/s11684018-0625-0

Jimenez, G., Blanch, A. R., Tamames, J., and Rossello-Mora, R. (2013). Complete genome sequence of Bacillus toyonensis BCT-7112T, the active ingredient of the feed additive preparation Toyocerin. Genome Announc. 1:e01080-13. doi: 10.1128/genomeA.01080-13

Jin, E., Liu, W., Ni, T., Ren, M., Zhou, J., Hu, Q., et al. (2017). Effects of boron on serum biochemical indexes and microstructure of immune organs in male obese rats. Wei Sheng Yan Jiu 46, 113-119. Chinese,

Jinglong, X., Xiaobin, L., Fang, Z., Chenchen, W., and Kailun, Y. (2019). Isolation and identification of an isoflavone reducing bacterium from feces from a pregnant horse. PLoS One 14:e0223503. doi: 10.1371/journal.pone.0223503

Kabeerdoss, J., Devi, R. S., Mary, R. R., Prabhavathi, D., Vidya, R., Mechenro, J., et al. (2011). Effect of yoghurt containing Bifidobacterium lactis Bb12(R) on faecal excretion of secretory immunoglobulin A and human beta-defensin 2 in healthy adult volunteers. Nutr. J. 10:138. doi: 10.1186/1475-2891-10-138

Kandel, S., Joubert, P., and Doty, S. (2017). Bacterial endophyte colonization and distribution within plants. Microorganisms 5:77. doi: 10.3390/microorganisms5 040077

Kantas, D., Papatsiros, V. G., Tassis, P. D., Giavasis, I., Bouki, P., and Tzika, E. D. (2015). A feed additive containing Bacillus toyonensis (Toyocerin $\left({ }^{\circledR}\right)$ ) protects against enteric pathogens in postweaning piglets. J. Appl. Microbiol. 118, 727-738. doi: 10.1111/jam.12729

Kechagia, M., Basoulis, D., Konstantopoulou, S., Dimitriadi, D., Gyftopoulou, K., Skarmoutsou, N., et al. (2013). Health benefits of probiotics: a review. Nutrients 2013:481651.

Khunajakr, N., Wongwicharn, A., Moonmangmee, D., and Tantipaiboonvut, S. (2008). Screening and identification of lactic acid bacteria producing antimicrobial compounds from pig gastrointestinal tracts. KMITL Sci. Technol. J. 8, 8-17.

Kong, C., Gao, R., Yan, X., Huang, L., and Qin, H. (2019). Probiotics improve gut microbiota dysbiosis in obese mice fed a high-fat or high-sucrose diet. Nutrition 60, 175-184. doi: 10.1016/j.nut.2018.10.002

Kumara, P. M., Shweta, S., Vasanthakumari, M. M., Sachin, N., Manjunatha, B. L., Jadhav, S. S., et al. (2014). "Endophytes and plant secondary metabolite synthesis: molecular and evolutionary perspective," in Advances in Endophytic Research, eds V. C. Verma and A. C. Gange (New Delhi.: Springer), 177-190. doi: 10.1007/978-81-322-1575-2_9

Lammers, A., Wieland, W. H., Kruijt, L., Jansma, A., Straetemans, T., Schots, A., et al. (2010). Successive immunoglobulin and cytokine expression in the small intestine of juvenile chicken. Dev. Comp. Immunol. 34, 1254-1262. doi: 10.1016/j.dci.2010.07.001

Li, J., Wu, H., Liu, Y., and Yang, L. (2020). High fat diet induced obesity model using four strains of mice: kunming, C57BL/6, BALB/c and ICR. Exp. Anim. 69, 326-335. doi: 10.1538/expanim.19-0148

Li, X., Wei, X., Sun, Y., Du, J., Li, X., Xun, Z., et al. (2019). High-fat diet promotes experimental colitis by inducing oxidative stress in the colon. Am. J. Physiol. Gastrointest. Liver Physiol. 317, G453-G462. doi: 10.1152/ajpgi.00103.2019

Lindner, C., Wahl, B., Fohse, L., Suerbaum, S., Macpherson, A. J., Prinz, I., et al. (2012). Age, microbiota, and T cells shape diverse individual IgA repertoires in the intestine. J. Exp. Med. 209, 365-377. doi: 10.1084/jem.20111980

Lv, M., Yu, B., Mao, X. B., Zheng, P., He, J., and Chen, D. W. (2012). Responses of growth performance and tryptophan metabolism to oxidative stress induced by diquat in weaned pigs. Animals 6, 928-934. doi: 10.1017/S1751731111002382

Miljkovic, M., Strahinic, I., Tolinacki, M., Zivkovic, M., Kojic, S., Golic, N., et al. (2015). AggLb is the largest cell-aggregation factor from Lactobacillus paracasei Subsp. paracasei BGNJ1-64, functions in collagen adhesion, and pathogen exclusion in vitro. PLoS One 10:e126387. doi: 10.1371/journal.pone.0126387

Mo, Q., Hu, L., Weng, J., Zhang, Y., Zhou, Y., Xu, R., et al. (2017). Euptox A induces G1 arrest and autophagy via p38 MAPK- and PI3K/Akt/mTORmediated pathways in mouse splenocytes. J Histochem. Cytochem. 65, 543-558. doi: 10.1369/0022155417722118

Mok, W. K., Tan, Y. X., Lyu, X. M., and Chen, W. N. (2020). Effects of submerged liquid fermentation of Bacillus subtilis WX-17 using okara as sole nutrient source on the composition of a potential probiotic beverage. Food Sci. Nutr. 8, 3119-3127. doi: 10.1002/fsn3.1541

Nematollahi, A., Sohrabvandi, S., Mortazavian, A. M., and Jazaeri, S. (2016). Viability of probiotic bacteria and some chemical and sensory characteristics in cornelian cherry juice during cold storage. Electron. J. Biotechnol. 21, 49-53.

Netto Candido, T. L., Bressan, J., and Alfenas, R. C. G. (2018). Dysbiosis and metabolic endotoxemia induced by high-fat diet. Nutr. Hosp. 35, 1432-1440. doi: $10.20960 /$ nh.1792

Nithya, V., and Halami, P. M. (2013). Evaluation of the probiotic characteristics of Bacillus species isolated from different food sources. Ann. Microbiol. 63, 129-137. doi: 10.1007/s13213-012-0453-4

Okyere, S. K., Mo, Q., Pei, G., Ren, Z., Deng, J., and Hu, Y. (2020). Euptox A induces G0 /GI arrest and apoptosis of hepatocyte via ROS, mitochondrial dysfunction and caspases-dependent pathways in vivo. J. Toxicol. Sci. 45, 661-671. doi: $10.2131 /$ jts. 45.661

Okyere, S. K., Wen, J., Cui, Y., Xie, L., Gao, P., Wang, J., et al. (2021). Toxic mechanisms and pharmacological properties of euptox A, a toxic monomer from A. adenophora. Fitoterapia 155:105032. doi: 10.1016/j.fitote.2021.105032

Ouyang, C. B., Liu, X. M., Liu, Q., Bai, J., Li, H. Y., Li, Y., et al. (2014). Toxicity assessment of cadinene sesquiterpenes from Eupatorium adenophorum in mice. Nat. Prod. Bioprospect. 5, 29-36. doi: 10.1007/s13659-014-0050-2

Özüsağlam, M. A., and Aksaray, U. (2010). Importance of Bacillus coagulans bacterium as probiotic in animal nutrition. SDU. J. Nat. Appl. Sci. 5, 50-57.

Pabari, K., Pithva, S., Kothari, C., Purama, R. K., Kondepudi, K. K., Vyas, B. R., et al. (2020). Evaluation of probiotic properties and prebiotic utilization potential of weissella paramesenteroides isolated from Fruits. Probiotics Antimicrob. Proteins 12, 1126-1138. doi: 10.1007/s12602-019-09630-w

Palm, N. W., de Zoete, M. R., Cullen, T. W., Barry, N. A., Stefanowski, J., Hao, L., et al. (2014). Immunoglobulin A coating identifies colitogenic bacteria in inflammatory bowel disease. Cell 158, 1000-1010. doi: 10.1016/j.cell.2014.08. 006

Pessoa, W. F. B., Melgaço, A. C. C., De Almeida, M. E., Ramos, L. P., Rezende, R. P., and Romano, C. C. (2017). In vitro activity of Lactobacilli with probiotic potential isolated from cocoa fermentation against Gardnerella vaginalis. BioMed. Res. Int. 2017, 1-10. doi: 10.1155/2017/3264194

Pizzino, G., Irrera, N., Cucinotta, M., Pallio, G., Mannino, F., Arcoraci, V., et al. (2017). Oxidative stress: harms and benefits for human health. Oxid. Med. Cell Longev. 2017:8416763. doi: 10.1155/2017/8416763

Ragul, K., Kandasamy, S., Devi, P. B., and Shetty, P. H. (2020). Safety and stability assessment of potential probiotic strains from fermented mango brine pickle. Probiotics Antimicrob. Proteins 12, 1039-1044. doi: 10.1007/s12602-01909617-7

Ren, Z., Gao, P., Okyere, S. K., Cui, Y., Wen, J., Jing, B., et al. (2021a). Ageratina adenophora inhibits spleen immune function in rats via the loss of the 
FRC Network and Th1-Th2 cell ratio elevation. Toxins 13:309. doi: 10.3390/ toxins 13050309

Ren, Z., Okyere, S. K., Wen, J., Xie, L., Cui, Y., Wang, S., et al. (2021b). An overview: the toxicity of Ageratina adenophora on animals and its possible interventions. Int. J. Mol. Sci. 22:11581. doi: 10.3390/ijms222111581

Ringø, E., Salinas, I., Olsen, R. E., Nyhaug, A., Myklebust, R., and Mayhew, T. M. (2007). Histological changes in intestine of Atlantic salmon (Salmo salar L.) following in vitro exposure to pathogenic and probiotic bacterial strains. Cell Tissue Res. 328, 109-116. doi: 10.1007/s00441-006-0323-0

Rojas-Solis, D., Vences-Guzmán, M. A., Sohlenkamp, C., and Santoyo, G. (2020). Bacillus toyonensis COPE52 modifies lipid and fatty acid composition, exhibits antifungal activity, and stimulates growth of tomato plants under saline conditions. Curr. Microbiol. 77, 2735-2744. doi: 10.1007/s00284-020-02069-1

Roos, T. B., de Moraes, C. M., Sturbelle, R. T., Dummer, L. A., Fischer, G., and Leite, F. P. L. (2018). Probiotics Bacillus toyonensis and Saccharomyces boulardii improve the vaccine immune response to Bovine herpesvirus type 5 in sheep. Res. Vet. Sci. 117, 260-265. doi: 10.1016/j.rvsc.2017.12.022

Segers, M. E., and Lebeer, S. (2014). Towards a better understanding of Lactobacillus rhamnosus GG - host interactions. Microb. Cell. Fact. 13:S7. doi: 10.1186/1475-2859-13-S1-S7

Simon, O. (2010). An interdisciplinary study on the mode of action of probiotics in pigs. J. Anim. Feed Sci. 19, 230-243. doi: 10.2527/2006.843608x

Sudan, S., Flick, R., Nong, L., and Li, J. (2021). Potential probiotic bacillus subtilis isolated from a novel niche exhibits broad range antibacte-rial activity and causes virulence and metabolic dysregulation in enterotoxic E. coli. Microorganisms 9:1483. doi: 10.3390/microorganisms9071483

Sun, W., Zeng, C., Liu, S., Fu, J., Hu, L., Shi, Z., et al. (2018). Ageratina adenophora induces mice hepatotoxicity via ROS-NLRP3-mediated pyroptosis. Sci. Rep. 8:16032. doi: 10.1038/s41598-018-34492-7

Talib, M., Yeap, H., Aziz, M., Masarudin, M. J., Sharifuddin, S. A., Hui, Y. W., et al. (2019). Isolation and characterization of Lactobacillus spp. from Kefir samples in Malaysia. Molecules 24:2606. doi: 10.3390/molecules24142606

Tambekar, D. H., and Bhutada, S. A. (2010). Studies on antimicrobial activity and characteristics of bacteriocins produced by Lactobacillus strains isolated from milk of domestic animals. Internet J. Microbiol. 8, 1-6.

Tan, B. L., and Norhaizan, M. E. (2019). Effect of high-fat diets on oxidative stress, cellular inflammatory response and cognitive function. Nutrients 11:2579. doi: 10.3390/nu11112579

Taras, D., Vahjen, W., Macha, M., and Simon, O. (2005). Response of performance characteristics and fecal consistency to long-lasting dietary supplementation with the probiotic strain Bacillus cereus var. toyoi to sows and piglets. Arch. Anim. Nutr. 59, 405-417. doi: 10.1080/17450390500353168

Touret, T., Oliveira, M., and Semedo-Lemsaddek, T. (2018). Putative probiotic lactic acid bacteria isolated from sauerkraut fermenta-tions. PLoS One 13:e0203501. doi: 10.1371/journal.pone.0203501

Unban, K., Kochasee, P., Shetty, K., and Khanongnuch, C. (2020). Tannin-tolerant and extracellular tannase producing bacillus isolated from traditional fermented tea leaves and their probiotic functional properties. Foods 9:490. doi: 10.3390/ foods 9040490

van der Heijden, R. A., Sheedfar, F., Morrison, M. C., Hommelberg, P. P., Kor, D., Kloosterhuis, N. J., et al. (2015). High-fat diet induced obesity primes inflammation in adipose tissue prior to liver in C57BL/6j mice. Aging (Albany NY) 7, 256-268. doi: 10.18632/aging.100738

Van Kaer, L., and Olivares-Villagomez, D. (2018). Development, homeostasis, and functions of intestinal intraepithelial lymphocytes. J. Immunol. 200, 2235-2244. doi: 10.4049/jimmunol.1701704

Van, L. A., Kroese, F. G., Visser, A., Nelis, G. F., Westerveld, B. D., Jansen, P. L., et al. (2004). Immunoglobulin coating of faecal bacteria in inflammatory bowel disease. Eur. J. Gastroenterol. Hepatol. 16, 669-674. doi: 10.1097/01.meg. 0000108346.41221 .19

Wagner, B. A., Buettner, G. R., and Burns, C. P. (1994). Free radical-mediated lipid peroxidation in cells: oxidizability is a function of cell lipid bisallylic hydrogen content. Biochemistry 33, 4449-4453.

Wang, K., Cao, G., Zhang, H., Li, Q., and Yang, C. (2019). Effects of Clostridium butyricum and Enterococcus faecalis on growth performance, immune function, intestinal morphology, volatile fatty acids, and intestinal flora in a piglet model. Food Funct. 10, 7844-7854. doi: 10.1039/c9fo01650c
Wang, W., Wang, Y., Hao, X., Duan, Y., Meng, Z., An, X., et al. (2020). Dietary fermented soybean meal replacement alleviates diarrhea in weaned piglets challenged with enterotoxigenic Escherichia coli K88 by modulating inflammatory cytokine levels and cecal microbiota composition. BMC Vet. Res. 16:245. doi: 10.1186/s12917-020-02466-5

Wu, Q. J., Liu, N., Wu, X. H., Wang, G. Y., and Lin, L. (2018). Glutamine alleviates heat stress-induced impairment of intestinal morphology, intestinal inflammatory response, and barrier integrity in broilers. Poult. Sci. 97, 26752683. doi: $10.3382 / \mathrm{ps} /$ pey123

Xin, J., Zeng, D., Wang, H., Sun, N., Zhao, Y., Dan, Y., et al. (2020). Probiotic Lactobacillus johnsonii BS15 promotes growth performance, intestinal immunity, and gut microbiota in piglets. Probiotics Antimicrob. Proteins 12, 184-193. doi: 10.1007/s12602-018-9511-y

Xu, C. M., Li, X. M., Qin, B. Z., and Liu, B. (2016). Effect of tight junction protein of intestinal epithelium and permeability of colonic mucosa in pathogenesis of injured colonic barrier during chronic recovery stage of rats with inflammatory bowel disease. Asian Pac. J. Trop. Med. 9, 148-152. doi: 10.1016/j.apjtm.2016. 01.001

Xu, R., Weng, J., Hu, L., Peng, G., Ren, Z., Deng, J., et al. (2018). Anti-NDV activity of 9-oxo10,11-dehydroageraphorone extracted from Eupatorium adenophorum Spreng in vitro. Nat. Prod. Res. 32, 2244-2247. doi: 10.1080/14786419.2017. 1371158

Yau, Y. F., El-Nezami, H., Galano, J. M., Kundi, Z. M., Durand, T., and Lee, J. C. (2020). Lactobacillus rhamnosus GG and oat beta-glucan regulated fatty acid profiles along the gut-liver-brain axis of mice fed with high fat diet and demonstrated antioxidant and anti-inflammatory potentials. Mol. Nutr. Food Res. 64:e2000566. doi: 10.1002/mnfr.202000566

Yoshizaki, K., Asai, M., and Hara, T. (2020). High-fat diet enhances working memory in the Y-Maze test in male C57BL/6J mice with less anxiety in the elevated plus maze test. Nutrients 12:2036. doi: 10.3390/nu1207 2036

Yu, Y., Lu, J., Oliphant, K., Gupta, N., Claud, K., and Lu, L. (2020). Maternal administration of probiotics promotes gut development in mouse off-springs. PLoS One 15:e0237182. doi: 10.1371/journal.pone.0237182

Zaved, H. K., Rahman, M. M., Rahman, A., Arafat, S. M. Y., and Rahman, M. S. (2008). Isolation and characterization of effective bacteria for solid waste degradation for organic manure. KMITL Sci. Technol. J. 8, $44-55$.

Zhang, K., Shi, S., and Han, W. (2018). Research progress in cytokines with chemokine-like function. Cell Mol. Immunol. 15, 660-662. doi: 10.1038/cmi. 2017.121

Zheng, J. D., He, Y., Yu, H. Y., Liu, Y. L., Ge, Y. X., Li, X. T., et al. (2019). Unconjugated bilirubin alleviates experimental ulcerative colitis by regulating intestinal barrier function and immune inflammation. World J. Gastroenterol 25, 1865-1878. doi: 10.3748/wjg.v25.i15.1865

Conflict of Interest: YH was employed by New Ruipeng Pet Healthcare Group Co., Ltd.

The remaining authors declare that the research was conducted in the absence of any commercial or financial relationships that could be construed as a potential conflict of interest.

Publisher's Note: All claims expressed in this article are solely those of the authors and do not necessarily represent those of their affiliated organizations, or those of the publisher, the editors and the reviewers. Any product that may be evaluated in this article, or claim that may be made by its manufacturer, is not guaranteed or endorsed by the publisher.

Copyright (c) 2022 Okyere, Wen, Cui, Xie, Gao, Zhang, Wang, Wang, Ran, Ren and Hu. This is an open-access article distributed under the terms of the Creative Commons Attribution License (CC BY). The use, distribution or reproduction in other forums is permitted, provided the original author(s) and the copyright owner(s) are credited and that the original publication in this journal is cited, in accordance with accepted academic practice. No use, distribution or reproduction is permitted which does not comply with these terms. 